\title{
Graph-based approaches to debugging and revision of terminologies in DL-Lite
}

\author{
Xuefeng Fu*, Guilin Qi, Yong Zhang, Zhangquan Zhou \\ School of Computer Science and Engineering, Southeast University, Nanjing, China
}

\begin{abstract}
In this paper, we deal with the problem of debugging and revision of incoherent terminologies. Ontology debugging aims to provide the explanation of the causes of incoherence and ontology revision aims to eliminate the incoherence. For this purpose, we propose the graph-based approaches to deal with the debugging and revision of terminologies for a family of lightweight ontology languages, DL-Lite. First of all, we transform DL-Lite ontologies to graphs. To deal with the problem of ontology debugging, we calculate the minimal incoherence-preserving subsets (MIPS) of an ontology by computing the minimal incoherence-preserving path-pairs (MIPP) based on the transformed graph. To deal with the problem of ontology revision, we propose the notion of revision state which separates the terminology of an ontology into two disjoint sets: the set of wanted axioms and the set of unwanted axioms. We further define a revision operator based on the revision state. Afterwards, two revision algorithms are proposed to instantiate the revision operator: one is based on a scoring function, and the other one is based on a hitting set tree. We implement these algorithms and conduct experiments of ontology debugging and ontology revision on several adapted real ontologies. The experimental results of ontology debugging show that our approach of calculating MIPS based on graph is efficient and outperforms the state of the art. The experimental results of ontology revision show that the algorithm based on a scoring function is more efficient than the algorithm based on a hitting set tree.
\end{abstract}

${ }^{*}$ Corresponding author

Email addresses: fxf@seu.edu.cn (Xuefeng Fu ), gqi@seu.edu.cn (Guilin Qi), zhangyong@seu.edu.cn (Yong Zhang), quanzz@seu.edu.cn (Zhangquan Zhou)

Preprint submitted to Knowledge-based systems

January 28, 2016

(C) 2016. This manuscript version is made available under the Elsevier user license

http://www.elsevier.com/open-access/userlicense/1.0/ 
Keywords:

DL-Lite, Ontology debugging, Ontology revision, Scoring function, Hitting set tree

\section{Introduction}

Ontologies play an important roles in semantic web and it is also important in recommender systems and social network [1]. However, the development and maintenance of ontologies are complex and error-prone. Thus, logical inconsistencies can easily occur in real ontologies [2]. Ontology debugging [3], which aims to pinpoint the causes of inconsistencies, and ontology revision [4], which aims to eliminate the inconsistencies, have become the key issues in ontology engineering.

Up to now, many approaches of ontology debugging are proposed to compute minimal unsatisfiability preserving subsets (MUPS) of an ontology $[3,5,6]$ or to compute minimal incoherence preserving subsets (MIPS) of an ontology $[5,7]$. MUPS is useful for relating a set of axioms to an unsatisfiable concept and MIPS is the minimal sub-TBox of $\mathcal{T}$ which is incoherent. It is argued that MIPS is more useful than MUPS when repairing an ontology, since every MIPS is a MUPS and removing one axiom from each MIPS can resolve the incoherence of an ontology.

Similarly, there also exist many approaches for ontology revision. Most works, such as those reported in [8], [9], on ontology revision are based on the AGM theory [10]. An important principle of these works is minimal change, i.e., a revision approach should delete information from the original ontology as little as possible. In [8], a kernel revision operator is proposed based on an incision function that is used to select axioms from each MIPS. In [9], the authors propose a revision operator based on a trust-based incision function which aims to find minimal set of axioms that need to be removed to render a terminology logically correct. Nevertheless, the delete operation may lead to the loss of valuable information in an ontology.

Recently, there is an increasing interest in inconsistency handling in DLLite, which is a family of tractable description logics. In this paper, we provide graph-based approaches to debug and to revise DL-Lite ontologies. At first, we encode an ontology into a graph, and derive subsumption relations between two concepts (or roles) by reachability of two nodes. Then, all MIPSs can be calculated by backtracking pairs of nodes in the graph. 
Thus this approach helps to avoid the computation of all MUPSs of the ontology w.r.t. all unsatisfiable concepts. Based on MIPS, we propose the notion of revision state, which separates the terminology of an ontology into two disjoint sets: the set of wanted axioms and the set of unwanted axioms. We further define a refinement revision operator based on the revision state. This operator aims to retrieve useful information from the set of unwanted axioms. Afterwards, we propose two revision algorithms to instantiate the revision operator: one is based on a scoring function, and the other one is based on a hitting set tree. We then evaluate the performance of these algorithms by conducting experiments on some (adapted) real ontologies. The experimental results of ontology debugging show that our approach of calculating MIPS based on graph is efficient and outperforms the state of the art. The experimental results of ontology revision show that the algorithm based on a scoring function is more efficient than the algorithm based on a hitting set tree.

The rest of the paper is organized as follows: We first introduce the theoretical basis of DL-Lite and a method of graph construction in Section 2. We then present our graph-based debugging and revision approaches in Section 3. In section 4, we present our algorithms to instantiate the revision operator. In section 5, we evaluate our algorithms using some adapted ontologies. We discuss related works in Section 6. Finally, we conclude this paper in Section 7 .

\section{Preliminaries}

\subsection{DL-Lite family}

In this subsection, we introduce DL-Lite $\mathcal{F \mathcal { R }}_{\mathcal{R}}$, which is an important language in DL-Lite that stands out for its tractable reasoning and efficient query answering [11] [12]. We start with the introduction of DL-Lite core $_{\text {, }}$ which is the core language for the DL-Lite family [13]. The complex con-

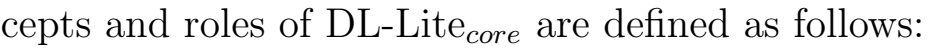

(1) $B \rightarrow A|\exists R,(2) R \rightarrow P| P^{-}$, (3) $C \rightarrow B \mid \neg B$, (4) $E \rightarrow R \mid \neg R$, where $A$ denotes an atomic concept, $P$ an atomic role, $B$ a basic concept, and $C$ a general concept. A basic concept can be either an atomic concept or a concept of the form $\exists R$, where $R$ denotes a basic role which can be either an atomic role or the inverse of an atomic role.

In DL-Lite core $_{\text {, an ontology }} \mathcal{O}=\langle\mathcal{T}, \mathcal{A}\rangle$ consists of a TBox $\mathcal{T}$ and an ABox $\mathcal{A}$, where $\mathcal{T}$ is a finite set of concept inclusion assertions of the form: 
$B \sqsubseteq C$; and $\mathcal{A}$ is a finite set of membership assertions of the form: $A(a)$, $P(a, b)$. In this paper, ABoxes will not be considered. DL-Lite $\mathcal{F}_{\mathcal{R}}$ extends DL-Lite $_{\text {core }}$ with inclusion assertions between roles which are the form $R \sqsubseteq E$ and functionality on roles (or on their inverses) which are the form (funct $R$ ) or $\left(\right.$ funct $\left.R^{-}\right)$. To keep the logic tractable, whenever a role inclusion $R_{1} \sqsubseteq R_{2}$ appears in $\mathcal{T}$, neither (funct $R_{2}$ ) nor (funct $R_{2}^{-}$) can appear in it.

The semantics of DL-Lite is defined by an interpretation $\mathcal{I}=\left(\triangle^{\mathcal{I}},{ }^{\mathcal{I}}\right)$ which consists of a non-empty domain set $\triangle^{\mathcal{I}}$ and an interpretation function . , which maps individuals, concepts and roles to elements of the domain, subsets of the domain and binary relations on the domain, respectively. The interpretation function can be extended to arbitrary concept (or role) descriptions and inclusion (or membership) assertions in a standard way [11]. Given an interpretation $\mathcal{I}$ and an assertion $\phi, \mathcal{I} \models \phi$ denotes that $\mathcal{I}$ satisfies $\phi$. An interpretation is called a model of an ontology $\mathcal{O}$, iff it satisfies each assertion in $\mathcal{O}$. An ontology $\mathcal{O}$ logically implies an assertion $\phi$, written $\mathcal{O}=\phi$, if all models of $\mathcal{O}$ satisfy $\phi$.

In DL-Lite, the assertions of the form $B_{1} \sqsubseteq B_{2}$ or $R_{1} \sqsubseteq R_{2}$ are called positive inclusions (PIs) and the assertions of the form $B_{1} \sqsubseteq \neg B_{2}$ or $R_{1} \sqsubseteq$ $\neg R_{2}$ are called negative inclusions (NIs). In order to take into account the interaction of inclusions, we introduce the definition of NI-closure [11].

Definition 1. Let $\mathcal{T}$ be a DL-Lite $\mathcal{F R}_{\mathcal{R}}$ TBox. We define the NI-closure of $\mathcal{T}$, denoted by $\operatorname{cln}(\mathcal{T})$, inductively as follows:

1. All negative inclusion assertions (NIs) in $\mathcal{T}$ are also in $\operatorname{cln}(\mathcal{T})$.

2. All functionality assertions in $\mathcal{T}$ are also in $\operatorname{cln}(\mathcal{T})$.

3. If $B_{1} \sqsubseteq B_{2}$ is in $\mathcal{T}$ and $B_{2} \sqsubseteq \neg B_{3}$ or $B_{3} \sqsubseteq \neg B_{2}$ is in $\operatorname{cln}(\mathcal{T})$, then also $B_{1} \sqsubseteq \neg B_{3}$ is in $\operatorname{cln}(\mathcal{T})$.

4. If $R_{1} \sqsubseteq R_{2}$ is in $\mathcal{T}$ and $\exists R_{2} \sqsubseteq \neg B$ or $B \sqsubseteq \neg \exists R_{2}$ is in $\operatorname{cln}(\mathcal{T})$, then also $\exists R_{1} \sqsubseteq \neg B$ is in $\operatorname{cln}(\mathcal{T})$.

5. If $R_{1} \sqsubseteq R_{2}$ is in $\mathcal{T}$ and $\exists R_{2}^{-} \sqsubseteq \neg B$ or $B \sqsubseteq \neg \exists R_{2}^{-}$is in $\operatorname{cln}(\mathcal{T})$, then also $\exists R_{1}^{-} \sqsubseteq \neg B$ is in $\operatorname{cln}(\mathcal{T})$.

6. If $R_{1} \sqsubseteq R_{2}$ is in $\mathcal{T}$ and $R_{2} \sqsubseteq \neg R_{3}$ or $R_{3} \sqsubseteq \neg R_{2}$ is in $\operatorname{cln}(\mathcal{T})$, then also $R_{1} \sqsubseteq \neg R_{3}$ is in $\operatorname{cln}(\mathcal{T})$.

7. If one of the assertions $\exists R \sqsubseteq \neg \exists R, \exists R^{-} \sqsubseteq \neg \exists R^{-}$and $R \sqsubseteq \neg R$ is in $\operatorname{cln}(\mathcal{T})$, then all three such assertions are in $\operatorname{cln}(\mathcal{T})$.

Definition 2. Let $\mathcal{T}$ be a DL-Lite $\mathcal{F R}_{\mathcal{R}}$ TBox. We define the PI-closure of $\mathcal{T}$, denoted by $\operatorname{clp}(\mathcal{T})$, inductively as follows: 
1. All positive inclusion assertions $(P I s)$ in $\mathcal{T}$ are also in $\operatorname{clp}(\mathcal{T})$.

2. If $B_{1} \sqsubseteq B_{2}$ is in $\operatorname{clp}(\mathcal{T})$ and $B_{2} \sqsubseteq B_{3}$ is in $\operatorname{clp}(\mathcal{T})$, then also $B_{1} \sqsubseteq B_{3}$ is in $\operatorname{clp}(\mathcal{T})$.

3. If $R_{1} \sqsubseteq R_{2}$ is in $\operatorname{clp}(\mathcal{T})$ and $R_{2} \sqsubseteq R_{3}$ is in $\operatorname{clp}(\mathcal{T})$, then also $R_{1} \sqsubseteq R_{3}$ is in $\operatorname{clp}(\mathcal{T})$.

4. If $R_{1} \sqsubseteq R_{2}^{-}$is in $\operatorname{clp}(\mathcal{T})$ and $R_{2} \sqsubseteq R_{3}^{-}$is in $\operatorname{clp}(\mathcal{T})$, then also $R_{1} \sqsubseteq R_{3}$ is in $\operatorname{clp}(\mathcal{T})$.

5. If $R_{1} \sqsubseteq R_{2}$ is in $\operatorname{clp}(\mathcal{T})$ and $\exists R_{2} \sqsubseteq B$ is in $\operatorname{clp}(\mathcal{T})$, then also $\exists R_{1} \sqsubseteq B$ is in $\operatorname{clp}(\mathcal{T})$.

6. If $R_{1} \sqsubseteq R_{2}$ is in $\operatorname{clp}(\mathcal{T})$ and $\exists R_{2}^{-} \sqsubseteq B$ is in $\operatorname{clp}(\mathcal{T})$, then also $\exists R_{1}^{-} \sqsubseteq B$ is in $\operatorname{clp}(\mathcal{T})$.

Finally, let $\operatorname{cl}(\mathcal{T})=\operatorname{clp}(\mathcal{T}) \cup \operatorname{cln}(\mathcal{T})$, where $\operatorname{cl}(\mathcal{T})$ is the closure of $\mathcal{T}$. We call a TBox $\mathcal{T}$ is closed if $\mathcal{T}=\operatorname{cl}(\mathcal{T})$.

\subsection{Logical conflict in TBox}

We now introduce several important notations closely related to ontology revision given in [14]. These notations will be used in the sequel.

Definition 3. Let $\mathcal{T}$ be a $D L$-Lite FR $_{\mathcal{R}}$ TBox. A concept $C$ (resp. role $R$ ) in $\mathcal{T}$ is unsatisfiable if and only if for each model $\mathcal{I}$ of $\mathcal{T}, C^{\mathcal{I}}=\emptyset\left(\right.$ resp. $\left.R^{\mathcal{I}}=\emptyset\right)$.

A TBox $\mathcal{T}$ is incoherent if and only if there exist at least one unsatisfiable concepts or roles in $\mathcal{T}$.

Definition 4. Let $\mathcal{T}$ be an incoherent TBox. A TBox $\mathcal{T}^{\prime} \subseteq \mathcal{T}$ is a minimal incoherence-preserving sub-TBox (MIPS) of $\mathcal{T}$ if and only if $\mathcal{T}^{\prime}$ is incoherent and every sub-TBox $\mathcal{T}^{\prime \prime} \subset \mathcal{T}^{\prime}$ is coherent.

Example 1. Given a TBox $\mathcal{T}$, where $\mathcal{T}=\{A \sqsubseteq B, B \sqsubseteq C, B \sqsubseteq D, D \sqsubseteq$ $\neg C, A \sqsubseteq \neg B\}$. According to Definition 3, $A$ and $B$ are unsatisfiable concepts. According to Definition 4, there are two MIPSs in $\mathcal{T}$, one is $\{A \sqsubseteq B, A \sqsubseteq$ $\neg B\}$, and the other is $\{B \sqsubseteq C, B \sqsubseteq D, D \sqsubseteq \neg C\}$.

Definition 5. Let $\mathcal{T}$ be a coherent DL-Lite FR $_{\text {R }}$ TBox. A TBox is logical closure of $\mathcal{T}$, denoted by $\mathrm{cn}(\mathcal{T})$, if $\phi \in \mathrm{cn}(\mathcal{T})$ for any $\mathcal{T} \models \phi$.

In the following, we give the relationship between $\operatorname{cl}(\mathcal{T})$ and $c n(\mathcal{T})$.

Theorem 1. Let $\mathcal{T}$ be a $D L-L i t e_{\mathcal{F R}} T B o x, \operatorname{cl}(\mathcal{T})=c n(\mathcal{T})$ if $\mathcal{T}$ is coherent 
Proof. According to the definition of $c n(\mathcal{T})$, it includes all axioms entailed by $\mathcal{T}$. Since for inductive rules in Definition 2 and Definition 1, all positive inclusion assertions, negative inclusion assertions and functionality assertions that can be entailed by $\mathcal{T}$, are included in $\operatorname{cl}(\mathcal{T})$. Therefore, $\operatorname{cl}(\mathcal{T}) \subseteq \operatorname{cn}(\mathcal{T})$.

Each axiom $\phi$ in a TBox of a DL-Lite ontology can have three forms: positive inclusion, negative inclusion and functionality assertions. To arbitrary axiom $\phi \in c n(\mathcal{T})$, we discuss the relationship between $c n(\mathcal{T})$ and $\operatorname{cl}(\mathcal{T})$ by considering these three forms respectively.

Let $\phi$ be a positive inclusion assertion of the form $A \sqsubseteq B$. Assuming that $\mathcal{I}$ is a model of $\mathcal{T}, \mathcal{I}$ satisfies axiom $A \sqsubseteq B$, that is $A^{\mathcal{I}} \subseteq B^{\mathcal{I}}$. Since $\mathcal{T}$ is coherent and $\phi \in c n(\mathcal{T})$ implies that $\mathcal{T} \vDash A \sqsubseteq B$, there exist concepts, without loss of generality, $B_{1}, \ldots, B_{n}$, such that $A^{\mathcal{I}} \subseteq B_{1}^{\mathcal{I}} \subseteq \ldots \subseteq B_{n}^{\mathcal{I}} \subseteq B^{\mathcal{I}}$. Then we can get that $\mathcal{T} \models A \sqsubseteq B_{1}, \mathcal{T} \models B_{1} \sqsubseteq B_{2}, \ldots, \mathcal{T} \models B_{n-1} \sqsubseteq B_{n}$, $\mathcal{T} \models B_{n} \sqsubseteq B$. According to the rules of definition of $\operatorname{clp}(\mathcal{T})$ which entail $A \sqsubseteq B$. Therefore, $\phi \in \operatorname{cl}(\mathcal{T})$.

Let $\phi$ be a negative inclusion assertion or a functionality assertion. $\phi \in$ $\operatorname{cn}(\mathcal{T})$ implies $\mathcal{T} \vDash \phi$. According to the Corollary 13 of [11], if $\mathcal{T} \vDash \phi$ then $\phi \in$ $\operatorname{cln}(T)$. Hence we can infer that $\phi \in \operatorname{cl}(\mathcal{T})$ because $\operatorname{cl}(\mathcal{T})=\operatorname{clp}(\mathcal{T}) \cup \operatorname{cln}(\mathcal{T})$.

Finally, we conclude that $\operatorname{cl}(\mathcal{T})=c n(\mathcal{T})$.

The coherence of TBox $\mathcal{T}$ is the prerequisite for $\operatorname{cl}(\mathcal{T})=c n(\mathcal{T})$. If TBox $\mathcal{T}$ is incoherent, $\mathcal{T}$ include at least one unsatisfiable concepts or roles. An unsatisfiable concept or role can infer an arbitrary consequence, so that the reasoning would turn out to be trivial. It can be shown in Example 2.

Example 2. Given a TBox $\mathcal{T}$, where $\mathcal{T}=\{A \sqsubseteq B, A \sqsubseteq \neg B, C \sqsubseteq D\}$. It is easy to check that $A$ is an unsatisfiable concept and $\mathcal{T}$ is incoherent. According to Definition 5, we can get $\operatorname{cn}(\mathcal{T})=\{A \sqsubseteq B, A \sqsubseteq \neg B, C \sqsubseteq$ $D, A \sqsubseteq \neg A, A \sqsubseteq C, A \sqsubseteq D\}$.

According to the definition of $\operatorname{cl}(\mathcal{T})$, we can get $\operatorname{cl}(\mathcal{T})=\{A \sqsubseteq B, A \sqsubseteq$ $\neg B, C \sqsubseteq D, A \sqsubseteq \neg A\}$.

\subsection{Graph construction}

Inspired by the work given in [15], [16] and [17], we transform a DL-Lite ontology into a graph. Based on the constructed graph, we can propose our debugging and revision approaches, which will be discussed in the next section. 
Let $\mathcal{T}$ be a DL-Lite TBox over a signature $\Sigma_{\mathcal{T}}$, containing symbols for atomic elements, i.e., atomic concept and atomic roles. the digraph $G_{\mathcal{T}}=$ $\langle N, E\rangle$ constructed from $\mathcal{T}$ over the signature $\Sigma_{\mathcal{T}}$ is given as follows:

1. for each concept $B$ in $\Sigma_{\mathcal{T}}, N$ contains the node $B$.

2. for each role $P$ in $\Sigma_{\mathcal{T}}, N$ contains the nodes $P, P^{-}, \exists P, \exists P^{-}$.

3. for each concept inclusion $B_{1} \sqsubseteq B_{2} \in \mathcal{T}, E$ contains the $\operatorname{arc}\left(B_{1}, B_{2}\right)$.

4. for each concept inclusion $B_{1} \sqsubseteq \neg B_{2} \in \mathcal{T}, E$ contains the $\operatorname{arc}\left(B_{1}, \neg B_{2}\right)$ and $N$ contains the node $\neg B_{2}$.

5. for each role inclusion $R_{1} \sqsubseteq R_{2} \in \mathcal{T}, E$ contains the $\operatorname{arc}\left(R_{1}, R_{2}\right), \operatorname{arc}\left(R_{1}^{-}, R_{2}^{-}\right)$, $\operatorname{arc}\left(\exists R_{1}, \exists R_{2}\right), \operatorname{arc}\left(\exists R_{1}^{-}, \exists R_{2}^{-}\right)$.

6. for each role inclusion $R_{1} \sqsubseteq \neg R_{2} \in \mathcal{T}, E$ contains the $\operatorname{arc}\left(R_{1}, \neg R_{2}\right)$, $\operatorname{arc}\left(R_{1}^{-}, \neg R_{2}^{-}\right), \operatorname{arc}\left(\exists R_{1}, \neg \exists R_{2}\right), \operatorname{arc}\left(\exists R_{1}^{-}, \neg \exists R_{2}^{-}\right)$and $N$ contains nodes $\neg R_{2}, \neg R_{2}^{-}, \neg \exists R_{2}, \neg \exists R_{2}^{-}$.

Based on (1)-(6), we construct a graph from a TBox $\mathcal{T}$. It has been shown in [16] that the problem of TBox classification in a DL-Lite ontology $\mathcal{O}$ can be done by computing the transitive closure of graph $G_{\mathcal{T}}$. For simplicity, we call these rules Construction Rules, and we use $C(R)$ to denote the node w.r.t. concept $C$ (role $R$ ).

In a graph built by Construction Rules, each node represents a basic concept or a basic role, while each arc represents an inclusion assertion, i.e. the start and end nodes are corresponding respectively to the left-hand and right-hand parts of an inclusion assertion. In order to ensure that the information represented in the TBox is preserved by the graph, we add nodes $R, R^{-}, \exists R, \exists R^{-}$for each role $R, \operatorname{arc}\left(R_{1}, R_{2}\right), \operatorname{arc}\left(R_{1}^{-}, R_{2}^{-}\right), \operatorname{arc}\left(\exists R_{1}, \exists R_{2}\right)$, $\operatorname{arc}\left(\exists R_{1}^{-}, \exists R_{2}^{-}\right)$for each role inclusion assertion $R_{1} \sqsubseteq R_{2}$.

\section{Graph-based debugging and revision}

In this section, we introduce our graph-based approaches for TBox debugging and revision. At first, we give theorems that serve as the theoretical basis for our approach.

\subsection{Theoretical basis}

To explain the equivalence of a DL-Lite TBox and its corresponding graph, we firstly introduce the theorem given in [16]. 
Theorem 2. Let $\mathcal{T}$ be a DL-Lite $\mathcal{F}_{\mathcal{R}}$ TBox that contains only positive inclusions and let $S_{1}$ and $S_{2}$ be two atomic concepts, or two atomic roles. $S_{1} \sqsubseteq S_{2}$ is entailed by $\mathcal{T}$ if and only if at least one of the following conditions holds:

1. a set $\mathcal{P}$ of positive inclusions exists in $\mathcal{T}$, such that $\mathcal{P} \models S_{1} \sqsubseteq S_{2}$

2. $\mathcal{T} \models S_{1} \sqsubseteq \neg S_{1}$

We can extend Theorem 2 to general concepts or roles, i.e., the subsumption relation may be a negative inclusion.

Theorem 3. Let $\mathcal{T}$ be a DL-Lite $\mathcal{F R}_{\mathcal{R}}$ TBox and let $S_{1}$ and $S_{2}$ be two atomic concepts, or two atomic roles. $S_{1} \sqsubseteq \neg S_{2}$ is entailed by $\mathcal{T}$ if and only if at least one of the following conditions holds:

1. a set $\mathcal{P}$ of positive inclusions and a negative inclusion $n \in \mathcal{T}$ exist in $\mathcal{T}$, such that $\mathcal{P} \cup\{n\} \models S_{1} \sqsubseteq \neg S_{2}$

2. $\mathcal{T} \models S_{1} \sqsubseteq \neg S_{1}$

Proof. $(\Leftarrow)$ This can be easily proved.

$(\Rightarrow)$ Assume $\mathcal{T} \models S_{1} \sqsubseteq \neg S_{2}$. Without loss of generality, we suppose that there exists a minimal axiom set $\mathcal{T}^{\prime}=\left\{\phi_{1}, \phi_{2}, \ldots, \phi_{n}\right\}$ in $\mathcal{T}$ and $\mathcal{T}^{\prime} \models S_{1} \sqsubseteq$ $\neg S_{2}$. According to Definition 1 and Definition 2, negative inclusions do not concur in the entailment of a set of positive inclusions. Therefore, there exists at least one negative inclusions in $\mathcal{T}^{\prime}$. Let $\phi_{i}$ be the first negative inclusion that occurs in the $\mathcal{T}^{\prime}$ and is of the form $B_{1} \sqsubseteq \neg B_{2}$, then the following cases are considered:

1. $\phi_{i}$ is the first negative inclusion of $\mathcal{T}^{\prime}$, then $\phi_{1}, \phi_{2}, \ldots, \phi_{i-1}$ are positive inclusions. If there has no negative inclusions in the $\phi_{i+1}, \phi_{i+2}, \ldots, \phi_{n}$, we can conclude that $\mathcal{T}^{\prime}$ only contains one negative inclusion $\phi_{i}$.

2. Suppose that there have more than one negative inclusions in $\phi_{i+1}, \phi_{i+2}$, $\ldots, \phi_{n}$, we select one negative inclusion and assume that it is of the form $B_{1}^{\prime} \sqsubseteq \neg B_{2}^{\prime}$. According to the syntax of DL-Lite [11], the general concept (role) only occurs on the right-hand side of inclusion assertions. Due to this constraint, inference can not be carried out between $\phi_{i}$ (we assume the form of $\phi_{i}$ is $B_{1} \sqsubseteq \neg B_{2}$ ) and $B_{1}^{\prime} \sqsubseteq \neg B_{2}^{\prime}$, so $\mathcal{T}^{\prime}$ is not a minimal set that entails $S_{1} \sqsubseteq \neg S_{2}$. Then we get a contradiction. 
Let $\mathcal{T}$ be a DL-Lite FR $_{\mathcal{R}}$ TBox and let $G_{\mathcal{T}}=\langle N, E\rangle$ be the digraph constructed from $\mathcal{T}$ according to the Construction Rules. We denote the transitive closure of $G_{\mathcal{T}}$ by $G_{\mathcal{T}}^{*}=\left\langle N, E^{*}\right\rangle$. According to Theorem 2 and Theorem 3, we can see that the entailment problem can be reduced to the graph reachability problem between concepts and roles. Consider for example, $\mathcal{T}=\left\{B_{1} \sqsubseteq B_{2}, B_{2} \sqsubseteq B_{3}\right\}$, whose corresponding graph $G$ contains two edges $\operatorname{arc}\left(B_{1}, B_{2}\right)$ and $\operatorname{arc}\left(B_{2}, B_{3}\right)$, it is obvious that $B_{1} \sqsubseteq B_{3} \in \operatorname{cl}(\mathcal{T})$ and $\operatorname{arc}\left(B_{1}, B_{3}\right)$ belongs to the transitive closure of $G$.

In order to prove the equivalence of an ontology and the graph constructed from it w.r.t. classification, we cite a theorem given in [16].

Theorem 4. Let $\mathcal{T}$ be a DL-Lite $\mathcal{F R}_{\mathcal{R}}$ TBox that contains only positive inclusions and let $G_{\mathcal{T}}=\langle N, E\rangle$ be its digraph representation. Let $S_{1}, S_{2}$ be two basic concepts (roles). $S_{1} \sqsubseteq S_{2} \in \operatorname{cl}(\mathcal{T})$ if and only if $\operatorname{arc}\left(S_{1}, S_{2}\right) \in E^{*}$.

Then we extend Theorem 4 as follows.

Theorem 5. Let $\mathcal{T}$ be a DL-Lite FR $_{\mathcal{R}}$ TBox and let $G_{\mathcal{T}}=\langle N, E\rangle$ be the digraph constructed from $\mathcal{T}$ according to the Construction Rules. Let $m$ be a basic concept (role) and $n$ be a general concept (role). $m \sqsubseteq n \in \operatorname{cl}(\mathcal{T})$ if and only if $\operatorname{arc}(m, n) \in E^{*}$.

Proof. $(\Leftarrow)$ If $\operatorname{arc}(m, n) \in E^{*}$, according to the definition of transitive closure of graph, there exists at least one paths starting from the node $m$ to the node $n$ in $G_{\mathcal{T}}$. Moreover, according to Construction Rules, for each edge in $G_{\mathcal{T}}$, it corresponds to at least one inclusion assertions in $\mathcal{T}$ or $\operatorname{cl}(\mathcal{T})$. We assume $\mathcal{S}$ is the set of these assertions. Obviously that $\mathcal{S} \models m \sqsubseteq n$. Therefore, $m \sqsubseteq n \in \operatorname{cl}(\mathcal{T})$.

$(\Rightarrow)$ If $m \sqsubseteq n \in \operatorname{cl}(\mathcal{T})$, we consider the following two cases:

1. If $m \sqsubseteq n \in c l p(\mathcal{T})$, we can infer $\operatorname{arc}(m, n) \in E^{*}$ based on Theorem 4 .

2. If $m \sqsubseteq n \in \operatorname{cln}(\mathcal{T})$, then $m \sqsubseteq n$ is in the form of $B_{1} \sqsubseteq \neg B_{2}$. According to Theorem 3, there exist a set of PIs and a NI $n$ such that $P \cup\{n\} \models$ $B_{1} \sqsubseteq \neg B_{2}$. Without loss of generality, we assume $n=\left\{B^{\prime} \sqsubseteq \neg B_{2}\right\}$ and $P \models B_{1} \sqsubseteq B^{\prime}$. According to Theorem 2, we can infer that $\operatorname{arc}\left(B_{1}, B^{\prime}\right) \in$ $E^{*}$. Therefore, $\operatorname{arc}\left(B_{1}, \neg B_{2}\right) \in E^{*}$. 


\subsection{TBox debugging}

In order to revise an incoherent TBox, we propose an approach for TBox debugging which provides the explanation of the incoherence of an TBox. In this subsection we present our graph-based approach for TBox debugging. At first, we define the notion of MIPP which corresponds to MIPS of an incoherent TBox.

Definition 6. Let $\mathcal{T}$ be a DL-Lite $e_{\mathcal{F} R}$ TBox and $G_{\mathcal{T}}=\langle N, E\rangle$ be the digraph constructed from $\mathcal{T}$ according to the Construction Rules. Then for arbitrary node $C \in N$, if there exist two paths that start from node $C$ to node $D$ and from node $C$ to node $\neg D$ respectively in $G_{\mathcal{T}}$ and there does not exist joint edge between path $C \rightarrow D$ and path $C \rightarrow \neg D$, we call these two paths as minimal incoherence-preserving path-pair (MIPP).

We use $\operatorname{mipp}\left(G_{\mathcal{T}}\right)$ to denote the set of all MIPPs in $G_{\mathcal{T}}$.

In the following, we show that the problem of computing MIPS of $\mathcal{T}$, denoted by mips $(\mathcal{T})$, can be reduced to that of finding MIPP in graph $G_{\mathcal{T}}$. Based on the correspondence between the syntax of DL-lite and the graph representation, the following theorem holds.

Theorem 6. Let $\mathcal{T}$ be a DL-Lite FR $_{\mathcal{R}}$ TBox, $\mathcal{T}$ is incoherent if and only if there exist at least one MIPP in $G_{\mathcal{T}}$.

Proof. $(\Leftarrow)$ If there exists a MIPP in $G_{\mathcal{T}}$, according to Definition 6 , we assume that there exist two paths, one is $C \rightarrow D$, and the other is $C \rightarrow \neg D$. In this case, we can infer both $C \sqsubseteq D$ and $C \sqsubseteq \neg D$ from $\mathcal{T}$ by Theorem 5 . Therefore, $\mathcal{T}$ contains at least one unsatisfiable concepts or roles.

$(\Rightarrow) \mathcal{T}$ is incoherent so that there will be at least one unsatisfiable concepts or roles in $\mathcal{T}$. Without loss of generality, suppose $C$ is an unsatisfiable concept and $C \sqsubseteq D \in \operatorname{cl}(\mathcal{T}), C \sqsubseteq \neg D \in \operatorname{cl}(\mathcal{T})$. According to Theorem 5, we have $\operatorname{arc}(C, D) \in G_{\mathcal{T}}$ and $\operatorname{arc}(C, \neg D) \in G_{\mathcal{T}}$. It is easy to see that there exist two paths without joint edge in the digraph $G_{\mathcal{T}}^{*}$, that is a MIPP by Definition 6.

We use $\mathcal{M}$ to denote the set of inclusion assertions in a path $P$ on the graph. It is obvious $\mathcal{M} \subseteq \operatorname{cl}(\mathcal{T})$. According to Theorem 3 and Theorem 5 , there will be a minimal subset $\mathcal{S} \subseteq \mathcal{T}$ such that $\mathcal{S}=\mathcal{M}$ and for every

$\mathcal{S}^{\prime} \subset \mathcal{S}, \mathcal{S}^{\prime} \not \models \mathcal{M}$. We call $\mathcal{S}$ as the minimal set in $\mathcal{T}$ corresponding to a path $P$, denoted by $\mathcal{S}_{\mathcal{T} \rightarrow P}$. Based on the notion, we discuss the relationship between MIPP and MIPS. 
Theorem 7. Let $\mathcal{T}$ be a DL-Lite ${ }_{\mathcal{F} R}$ TBox and $\mathcal{S}_{\mathcal{T} \rightarrow M I P P}$ be the minimal set in $\mathcal{T}$ corresponding to a MIPP in $G_{\mathcal{T}}$. Then $\mathcal{S}_{\mathcal{T} \rightarrow \text { MIPP }}$ is a MIPS in $\mathcal{T}$.

Proof. By Theorem 6, it is easy to see that $\mathcal{T}$ is incoherent. Let $\mathcal{M}$ be the set of inclusion assertions corresponding to the MIPP. For any $\mathcal{S}^{\prime} \subset$ $\mathcal{S}_{\mathcal{T} \rightarrow \text { MIPP }}, \mathcal{S}^{\prime} \not \models \mathcal{M}$ because $\mathcal{S}_{\mathcal{T} \rightarrow \text { MIPP }}$ is the minimal subset of $\mathcal{T}$ that satisfies $\mathcal{S}_{\mathcal{T} \rightarrow \text { MIPP }} \models \mathcal{M}$. According to Construction Rules, there does not exist a MIPP in $G_{\mathcal{S}^{\prime}}$. Then $\mathcal{S}_{\mathcal{T} \rightarrow M I P P}$ is incoherent and $\mathcal{S}^{\prime}$ is coherent. According to Definition $4, \mathcal{S}_{\mathcal{T} \rightarrow M I P P}$ is a MIPS in $\mathcal{T}$.

Theorem 8. Let $\mathcal{T}$ be a DL-Lite F $_{\mathcal{R}}$ TBox. The set of all $\mathcal{S}_{\mathcal{T} \rightarrow M I P P}$ in $G_{\mathcal{T}}$ corresponds to the set of all MIPS in $\mathcal{T}$.

Proof. For any MIPP in $\operatorname{mipp}\left(G_{\mathcal{T}}\right)$, by Theorem $7, \mathcal{S}_{\mathcal{T} \rightarrow M I P P} \in \operatorname{mips}(\mathcal{T})$. For any MIPS $\mathcal{M}$ in mips $(\mathcal{T}), \mathcal{M}$ is incoherent and for any $\mathcal{M}^{\prime} \subset \mathcal{M}, \mathcal{M}^{\prime}$ is coherent. Therefore, there exists a MIPP in $G_{\mathcal{M}}$ and it does not exist in $G_{\mathcal{M}^{\prime}}$. It is easy to see that $\mathcal{M}$ corresponds to the MIPP in $G_{\mathcal{T}}$ and $\mathcal{M}$ is contained in the set of all $\mathcal{S}_{\mathcal{T} \rightarrow \text { MIPP }}$ corresponding to MIPP in $\mathcal{T}$.

The case of role $R$ can be proved analogously.

\subsection{TBox revision}

TBox debugging only provides the cause of incoherence in a TBox. We now consider TBox revision which helps to further eliminate the conflicts based on the result of debugging.

In our work, we restrict to the case that $\mathcal{T}_{1}$ and $\mathcal{T}_{2}$ are coherent and $\mathcal{T}_{1} \cup \mathcal{T}_{2}$ is incoherent. We consider extending the definition of MIPS given in [8].

Definition 7. Let $\mathcal{T}_{1}$ and $\mathcal{T}_{2}$ be two coherent DL-Lite $\mathcal{F}_{\mathcal{R}}$ TBoxes and $\mathcal{T}_{1} \cup$ $\mathcal{T}_{2}$ is incoherent. A minimal incoherence-preserving sub-TBox of $\mathcal{T}_{1}$ w.r.t. $\mathcal{T}_{2}$, denoted by $\operatorname{MIPS}_{\mathcal{T}_{2}}\left(\mathcal{T}_{1}\right)$, is a sub-TBox of $\mathcal{T}_{1}$ which satisfies both of the following conditions:

1. $M I P S_{\mathcal{T}_{2}}\left(\mathcal{T}_{1}\right) \cup \mathcal{T}_{2}$ is incoherent.

2. $\forall \mathcal{T}_{1}^{\prime} \subset M I P S_{\mathcal{T}_{2}}\left(\mathcal{T}_{1}\right), \mathcal{T}_{1}^{\prime} \cup \mathcal{T}_{2}$ is coherent.

We denote the set of all MIPS of $\mathcal{T}_{1}$ w.r.t $\mathcal{T}_{2}$ by mips $\operatorname{T}_{\mathcal{T}_{2}}\left(\mathcal{T}_{1}\right)$

Definition 7 can be applied to a single TBox. In this case, an incoherent TBox can be divided into a static part and a rebuttal part. The rebuttal part consists of the axioms in MIPS. In the following, we present a modified notation of revision state which was proposed in [18]. 
Definition 8. Let $\mathcal{T}_{1}$ and $\mathcal{T}_{2}$ be two DL-Lite $\mathcal{F}_{\mathcal{R}}$ TBoxes and $\mathcal{T}=\mathcal{T}_{1} \cup \mathcal{T}_{2}$. The revision state of $\mathcal{T}$ is a tuple $\left(\mathcal{T}, \mathcal{T}_{\vDash}, \mathcal{T}_{\models}\right)$ that satisfies the following conditions:

1. $\mathcal{T}_{\vDash} \subseteq \mathcal{T}, \mathcal{T}_{\not} \subseteq \mathcal{T}_{1}$

2. $\mathcal{T}_{\vDash} \cap \mathcal{T}_{\not}=\emptyset$.

A revision state is complete, if $\mathcal{T}=\mathcal{T}_{\vDash} \cup \mathcal{T}_{\sharp}$, and incomplete otherwise. $A$ revision state is coherent if there is no $\phi \in \mathcal{T}_{\not}$ such that $\mathcal{T}_{\vDash} \vDash \phi$.

A revision state actually separates a TBox into two parts: $\mathcal{T}_{\vDash}$, which includes wanted inclusion assertions, and $\mathcal{T}_{\sharp}$, which contains unwanted inclusion assertions. In a complete revision state, we can remove axioms in unwanted set $\mathcal{T}_{\not}$ from $\mathcal{T}$ to accomplish the purpose of revision. The following example illustrate the idea.

Example 3. Let $\mathcal{T}_{1}=\{A \sqsubseteq \exists S, \exists S \sqsubseteq E\}, \mathcal{T}_{2}=\{\exists R \sqsubseteq X, A \sqsubseteq \neg X, S \sqsubseteq R\}$. $\mathcal{T}=\mathcal{T}_{1} \cup \mathcal{T}_{2}$. It is easy to check that concept $A$ is an unsatisfiable concept in $\mathcal{T}$, and we get a complete revision state of $\mathcal{T}$ given as follows.

1. $\mathcal{T}_{\vDash}=\{\exists S \sqsubseteq E, \exists R \sqsubseteq X, A \sqsubseteq \neg X, S \sqsubseteq R\}$.

2. $\mathcal{T}_{\leftleftarrows}=\{A \sqsubseteq \exists S\}$.

We can simply revise the merged TBox by removing $\mathcal{T}_{\not}$. However, the naive revision method may lose too much information in the original TBox. In example 3 , we can see that $A \sqsubseteq E \in \operatorname{cl}(\mathcal{T})$ and $A \sqsubseteq E \notin \operatorname{cl}\left(\mathcal{T}_{F}\right)$. Nevertheless, $\{A \sqsubseteq E\} \cup \mathcal{T}_{\mathbb{F}}$ is coherent, which means the inclusion assertion $\{A \sqsubseteq E\}$ can be preserved. In order to avoid the loss of information, we define the refinement of revision state.

Definition 9. Let $\mathcal{T}_{1}$ and $\mathcal{T}_{2}$ be two DL-Lite $e_{\mathcal{F}}$ TBoxes, $\mathcal{T}=\mathcal{T}_{1} \cup \mathcal{T}_{2}$.A coherent refinement of revision state $\left(\mathcal{T}, \mathcal{T}_{F}, \mathcal{T}_{F}\right)$ is $\left(\mathcal{T}, \mathcal{T}_{\vDash} \cup \mathcal{T}_{\vDash}^{r}, \mathcal{T}_{\sharp}\right)$, where $\mathcal{T}_{\vDash}^{r}=\left\{\psi \in \operatorname{cl}_{\mathcal{T}}(\phi) \mid \mathcal{T}_{\vDash} \cup\{\psi\}\right.$ is coherent, $\left.\phi \in \mathcal{T}_{\not}\right\}$.

In the above definition, $\operatorname{cl}_{\mathcal{T}}(\phi)$ denotes the closure of inclusion assertion $\phi$ w.r.t $\mathcal{T}$. Referring to the previous rules in Definition 2 and Definition 1, we give inductive rules for calculating $c l_{\mathcal{T}}(\phi)$. At first, inclusion assertions $\phi$ is in $\operatorname{cl}_{\mathcal{T}}(\phi)$. If $B_{1} \sqsubseteq B_{2}$ is in $\operatorname{cl}_{\mathcal{T}}(\phi)$ and $B_{2} \sqsubseteq B_{3}$ is in $\mathcal{T}$, then $B_{1} \sqsubseteq B_{3}$ is in $c l_{\mathcal{T}}(\phi)$. Analogously, other rules can be defined.

When incorporating a new TBox into an old one, refinement of revision state can retain original information as much as possible by adding some 
axioms into $\mathcal{T}_{\vDash}$. According to Definition 9, in Example 3, we can obtain $\mathcal{T}_{\vDash}^{r}=\{A \sqsubseteq E\}$.

In general, there could be more than one revision states. A problem is that which axioms are unwanted. Therefore, we propose some principles to address this problem.

In the revision process, a natural requirement is that the operator should follows the principle of minimal of changes [4], which says that a revision should result in a minimal modification. Inspired by the work in [19], we give the definition of fewer change to formalize the above principle.

Definition 10. Let $\mathcal{T}_{1}$ and $\mathcal{T}_{2}$ be two DL-Lite ${ }_{F R}$ TBoxes, $\mathcal{T}=\mathcal{T}_{1} \cup \mathcal{T}_{2}$ and $R S=\left(\mathcal{T}, \mathcal{T}_{F}, \mathcal{T}_{\models}\right)$ and $R S^{\prime}=\left(\mathcal{T}, \mathcal{T}_{\models}^{\prime}, \mathcal{T}_{\not \prime}^{\prime}\right)$ be two refinement of revision states w.r.t $\mathcal{T} . R S^{\prime}$ has fewer changes than $R S$ if

1. $\left|\mathcal{T}_{\not \neq}^{\prime}\right|<\left|\mathcal{T}_{\not \neq}\right|$, or

2. $\left|\mathcal{T}_{\not \models}^{\prime}\right|=\left|\mathcal{T}_{\not \models}\right|$, and $\left|\mathcal{T}_{\models}^{\prime}\right|<\left|\mathcal{T}_{\vDash}\right|$.

In the above definition, , $R S^{\prime}$ has fewer changes than $R S$ means $R S^{\prime}$ has fewer unwanted axioms than $R S$, or if the number of axioms of $R S^{\prime}$ has the same as $R S$, then the number of axioms of $R S^{\prime}$ should be less than that of $R S$.

This definition gives the conditions of selecting axioms when calculating refinement of revision state. In order to satisfy the condition of fewer changes, we give a revision function which is actually a composite of two parts. The first part calculates the unwanted axioms of TBox, and the second part refines valuable information from the results of the first part. The first part of our revision function can be implemented by an incision function [8].

Definition 11. Let $\mathcal{T}_{1}$ and $\mathcal{T}_{2}$ be two DL-Lite F $_{\mathcal{R}}$ TBoxes and $\mathcal{T}=\mathcal{T}_{1} \cup \mathcal{T}_{2}$. An incision function for $\mathcal{T}$, denoted by $\sigma$, is a function $\left(\sigma: 2^{2^{\mathcal{T}}} \rightarrow 2^{\mathcal{T}}\right)$ such that

1. $\sigma\left(\operatorname{mips}_{\mathcal{T}_{2}}\left(\mathcal{T}_{1}\right)\right) \subseteq \bigcup_{\mathcal{T}_{i} \in \text { mips }_{\mathcal{T}_{2}}\left(\mathcal{T}_{1}\right)} \mathcal{T}_{i}$

2. if $\mathcal{T}^{\prime} \in \operatorname{mips}_{\mathcal{T}_{2}}\left(\mathcal{T}_{1}\right)$, then $\mathcal{T}^{\prime} \cap \sigma\left(\operatorname{mips}_{\mathcal{T}_{2}}\left(\mathcal{T}_{1}\right)\right) \neq \emptyset$.

An incision function selects at least one axioms from each non-empty $\operatorname{MIPS}_{\mathcal{T}_{2}}\left(\mathcal{T}_{1}\right)$. To meet the conditions of fewer changes, the results should be minimal, that is, no other incision function $\sigma^{\prime}$ satisfies $\sigma^{\prime}\left(\operatorname{mips}_{\mathcal{T}_{2}}\left(\mathcal{T}_{1}\right)\right) \subset$ $\sigma\left(\operatorname{mips}_{\mathcal{T}_{2}}\left(\mathcal{T}_{1}\right)\right)$.

In the second part of the revision function, we propose a refinement function which expands the results of incision function $\sigma$. We define the refinement function in the following way: 
Definition 12. Let $\mathcal{T}_{1}$ and $\mathcal{T}_{2}$ be two DL-Lite FR $_{\mathcal{R}}$ TBoxes and $\mathcal{T}=\mathcal{T}_{1} \cup \mathcal{T}_{2}$. A refinement function for $\mathcal{T}$, denoted by $\xi$, is a function $\left(\xi: 2^{2^{\mathcal{T}}} \rightarrow 2^{\mathcal{T}}\right)$ based on incision function $\sigma$ such that,

1. $\xi\left(\operatorname{mips}_{\mathcal{T}_{2}}\left(\mathcal{T}_{1}\right)\right) \subseteq \operatorname{cl}_{\mathcal{T}}(\phi), \phi \in \sigma\left(\operatorname{mips}_{\mathcal{T}_{2}}\left(\mathcal{T}_{1}\right)\right)$,

2. $\xi\left(\right.$ mips $\left._{\mathcal{T}_{2}}\left(\mathcal{T}_{1}\right)\right) \cup \mathcal{T}_{2}$ is coherent.

According to Definition 11 and Definition 12, we obtain a revision function by composing an incision function and a refinement function, denoted by $\mathcal{F}_{r}=\sigma \oplus \xi$.

In the composite function $\mathcal{F}_{r}=\sigma \oplus \xi$, incision function $\sigma$ selects unwanted axioms from MIPS and refinement function $\xi$ refines result of $\sigma$. However, among different types of revision functions, some of them cannot satisfy the conditions of fewer change. To avoid the above case, we give the following definition.

Definition 13. Let $\mathcal{T}_{1}$ and $\mathcal{T}_{2}$ be two DL-Lite FR $_{\mathcal{R}}$ TBoxes and $\mathcal{T}=\mathcal{T}_{1} \cup \mathcal{T}_{2}$. A revision function $\mathcal{F}_{r}=\sigma \oplus \xi$ for $\mathcal{T}$ is optimal with respect to a lexicographic order if there is no other revision function $\mathcal{F}_{r}^{\prime}=\sigma^{\prime} \oplus \xi^{\prime}$ that satisfies the following conditions:

1. $\left.\mid \sigma^{\prime}\left(\operatorname{mips}_{\mathcal{T}_{2}}\left(\mathcal{T}_{1}\right)\right)\right)|<| \sigma\left(\operatorname{mips}_{\mathcal{T}_{2}}\left(\mathcal{T}_{1}\right)\right) \mid$

2. if $\left.\mid \sigma^{\prime}\left(\operatorname{mips}_{\mathcal{T}_{2}}\left(\mathcal{T}_{1}\right)\right)\right)|=| \sigma\left(\operatorname{mips}_{\mathcal{T}_{2}}\left(\mathcal{T}_{1}\right)\right) \mid$, then $\left|\xi^{\prime}\left(\operatorname{mips}_{\mathcal{T}_{2}}\left(\mathcal{T}_{1}\right)\right)\right|<\mid \xi\left(\right.$ mips $\left._{\mathcal{T}_{2}}\left(\mathcal{T}_{1}\right)\right) \mid$

Let $\left(\mathcal{T}, \mathcal{T}_{\vDash} \cup \mathcal{T}_{E}^{r}, \mathcal{T}_{F}\right)$ be a refinement revision state of $\mathcal{T}=\mathcal{T}_{1} \cup \mathcal{T}_{2}$. We obtain that $\mathcal{T}_{\sharp}=\sigma\left(\operatorname{mips}_{\mathcal{T}_{2}}\left(\mathcal{T}_{1}\right)\right), \mathcal{T}_{\vDash}^{r}=\xi\left(\operatorname{mips}_{\mathcal{T}_{2}}\left(\mathcal{T}_{1}\right)\right)$ and $\mathcal{T}_{\vDash}=\left(\mathcal{T} \backslash \mathcal{T}_{\sharp}\right)$.

Based on the above discussion, we give the definition of an operator for revising a TBox $\mathcal{T}_{1}$ with a newly received TBox $\mathcal{T}_{2}$. The idea is that we obtain the revision state of the merged TBox by calculating $\operatorname{mips}_{\mathcal{T}_{2}}\left(\mathcal{T}_{1}\right)$.

Definition 14. Let $\mathcal{T}_{1}$ and $\mathcal{T}_{2}$ be two TBoxes and $\mathcal{T}=\mathcal{T}_{1} \cup \mathcal{T}_{2}$. Let $\left(\mathcal{T}, \mathcal{T}_{F}, \mathcal{T}_{F}\right)$ be a revision state of $\mathcal{T}$ and $\left(\mathcal{T}, \mathcal{T}_{\equiv} \cup \mathcal{T}_{E}^{r}, \mathcal{T}_{\neq}^{r}\right)$ be the refinement revision state of $\mathcal{T}$. The refinement revision operator $\mathrm{o}_{\mathrm{F}}$ is defined as follows: $\mathcal{T}_{1} \mathrm{o}_{\mathrm{F}} \mathcal{T}_{2}=$ $\left(\mathcal{T}_{1} \backslash \mathcal{T}_{\not \neq}^{r}\right) \cup \mathcal{T}_{\vDash}^{r} \cup \mathcal{T}_{2}$

In the following, we discuss the properties of the refinement revision operator.

Proposition 1. Let $\mathcal{T}_{1}$ and $\mathcal{T}_{2}$ be two TBoxes. The operator $o_{F}$ satisfies the following properties:

R1. $\mathcal{T}_{2} \subseteq \mathcal{T}_{1}$ of $\mathcal{T}_{2}$. 
R2. if $\mathcal{T}_{1} \cup \mathcal{T}_{2}$ is coherent, then $\mathcal{T}_{1} \circ_{\vDash} \mathcal{T}_{2}=\mathcal{T}_{1} \cup \mathcal{T}_{2}$.

R3. if $\mathcal{T}_{2}$ is coherent, then $\mathcal{T}_{1} \circ \mathrm{O}_{F} \mathcal{T}_{2}$ is coherent.

R4. if $\mathcal{T}_{2} \equiv \mathcal{T}_{2}^{\prime}$, then $\mathcal{T}_{1}$ of $\mathcal{T}_{2} \equiv \mathcal{T}_{1}$ of $\mathcal{T}_{2}^{\prime}$.

R5. if $\phi \in \mathcal{T}_{1}$ and $\phi \notin \mathcal{T}_{1} \mathrm{o}_{\vDash} \mathcal{T}_{2}$, then there exists $\mathcal{S}_{1} \subseteq \mathcal{T}_{1}$ such that $\mathcal{S}_{1} \cup \mathcal{T}_{2}$ is coherent, but $\mathcal{S}_{1} \cup \mathcal{T}_{2} \cup\{\phi\}$ is not.

Proof. According to Definition 14, $\mathcal{T}_{2} \subseteq\left(\mathcal{T}_{1} \backslash \mathcal{T}_{\neq}^{r}\right) \cup \mathcal{T}_{F}^{r} \cup \mathcal{T}_{2}=\mathcal{T}_{1} \circ{ }_{F} \mathcal{T}_{2}$. It implies that (R1) holds. If $\mathcal{T}_{1} \cup \mathcal{T}_{2}$ is coherent, we can get $\operatorname{mips}_{\mathcal{T}_{2}}\left(\mathcal{T}_{1}\right)=\emptyset$, which indicates that $\mathcal{T}_{\vDash}$ and $\mathcal{T}_{\vDash}$ in a refinement revision state are empty set. It implies that (R2) holds. According to Definition 14, it is clear that (R3) holds. If $\mathcal{T}_{2} \equiv \mathcal{T}_{2}^{\prime}$, we can get mips $\mathcal{T}_{2}\left(\mathcal{T}_{1}\right)=\operatorname{mips}_{\mathcal{T}_{2}^{\prime}}\left(\mathcal{T}_{1}\right)$ according to Definition 7. Thus, $\sigma\left(\operatorname{mips}_{\mathcal{T}_{2}}\left(\mathcal{T}_{1}\right)\right)=\sigma\left(\operatorname{mips}_{\mathcal{T}_{2}^{\prime}}\left(\mathcal{T}_{1}\right)\right)$. It implies that $(\mathrm{R} 4)$ holds. According to Definition 14, the prerequisite of (R5) indicates that $\phi \in \mathcal{T}_{1} \backslash\left(\mathcal{T}_{1} \circ_{\vDash} \mathcal{T}_{2}\right) \subseteq \mathcal{T}_{\not \nvdash}$. In the previous discussion, we know that $\mathcal{T}_{\not}$ is the set of axioms selected by the incision function. Therefore, axiom $\phi$ satisfied the prerequisite of (R5) must be in a subset of $\mathcal{T}_{1}$ which is in conflict with $\mathcal{T}_{2}$. It is implies that (R5) holds.

\section{Graph-based algorithms for TBox debugging and revision}

In this section, we present our graph-based algorithms for TBox debugging and revision. In the process of debugging, all MIPPs, which can be transformed to MIPSs of ontology, can be calculated based on $G_{\mathcal{T}}$. In the process of revision, the incoherence of ontology can be eliminated based on the MIPPs calculated in the process of debugging.

\subsection{Graph-based algorithm for TBox debugging}

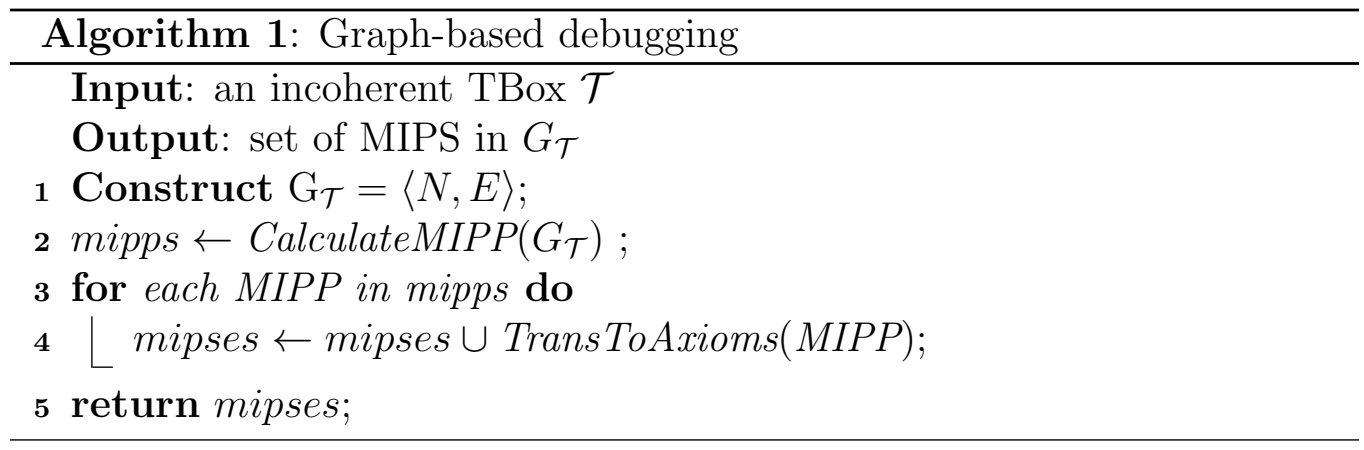


We propose Algorithm 1 for calculating all MIPSs of a TBox. We then give an example in the following to illustrate the procedure of this algorithm.

Let $S$ be a concept or a role expression. At the beginning of Algorithm 1, the TBox will be transformed into a graph in line 1, and then Algorithm 2 will be invoked to calculate MIPPs. In lines 2-6 of Algorithm 2, descendant nodes of $S$ and $\neg S$ can be obtained. If there is a node (called $S^{\prime}$ ) between descendant nodes of node $S$ (specially, it includes $S$ itself for the case $S \sqsubseteq$ $\neg S)$ and those of node $\neg S$. This also means that there exists a path-pair $\left\langle S^{\prime} \rightarrow S, S^{\prime} \rightarrow \neg S\right\rangle$ on $G_{\mathcal{T}}$. Since $S^{\prime} \rightarrow S$ and $S^{\prime} \rightarrow \neg S$ have no joint edge, $\left\langle S^{\prime} \rightarrow S, S^{\prime} \rightarrow \neg S\right\rangle$ is a MIPP.

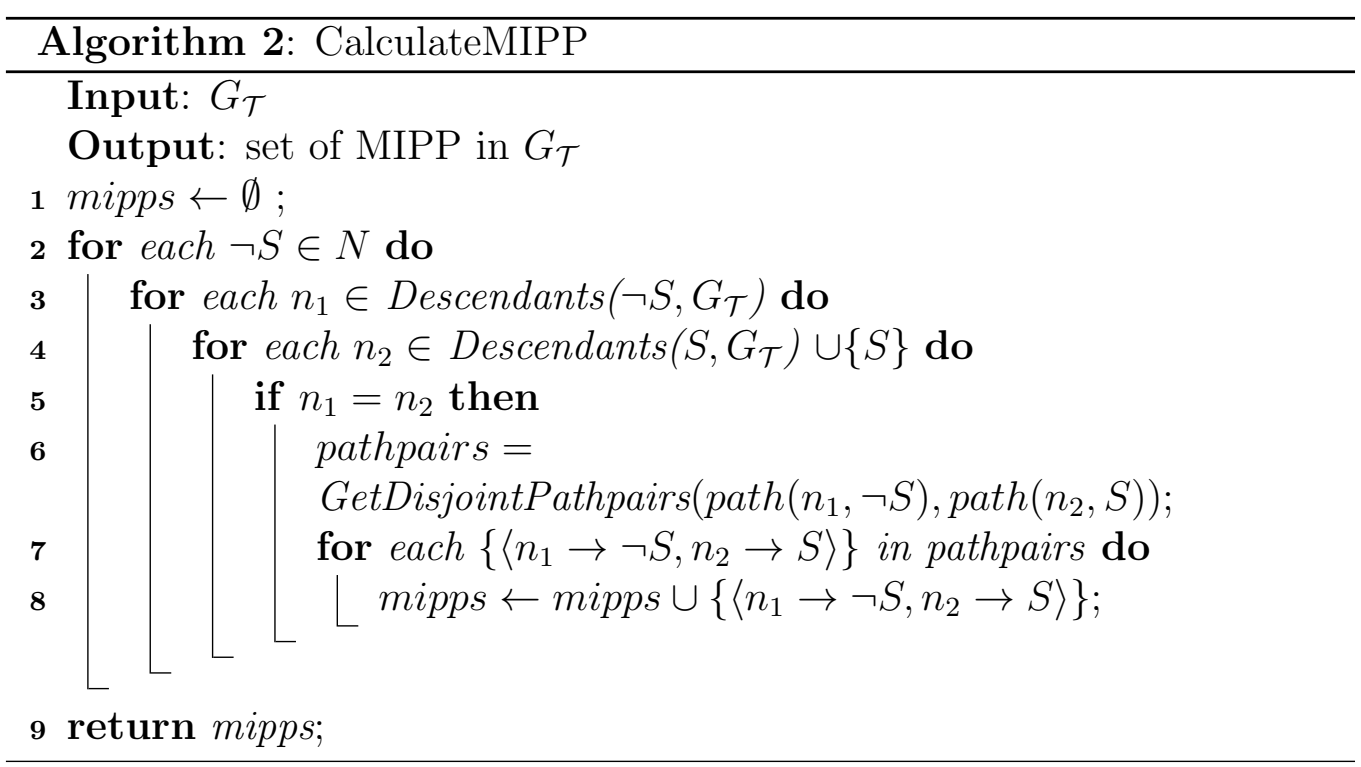

The function GetDisjointPathpairs is applied to calculate path-pairs between two disjoint nodes, and there is no intersection edge between these path-pairs. Algorithm 3 is applied to transform a MIPP into a MIPS.

In the following, we first discuss the complexity of Algorithm 1, Algorithm 2 and Algorithm3. According to the construction rules of graph, line 1 of Algorithm 1 can be executed in polynomial time. In line 2 of Algorithm 1, Algorithm 2 (CalculateMIPP) is invoked. Thus, we turn to discuss the complexity of Algorithm 2.

In line 2 of Algorithm 2 , all the negative nodes of $G_{\mathcal{T}}$ can be accessed in the first loop. In lines 3-4 of Algorithm 2, the descendant nodes of each negative node $\neg A$ and its disjoint node $A$ can be calculated. At the end of 


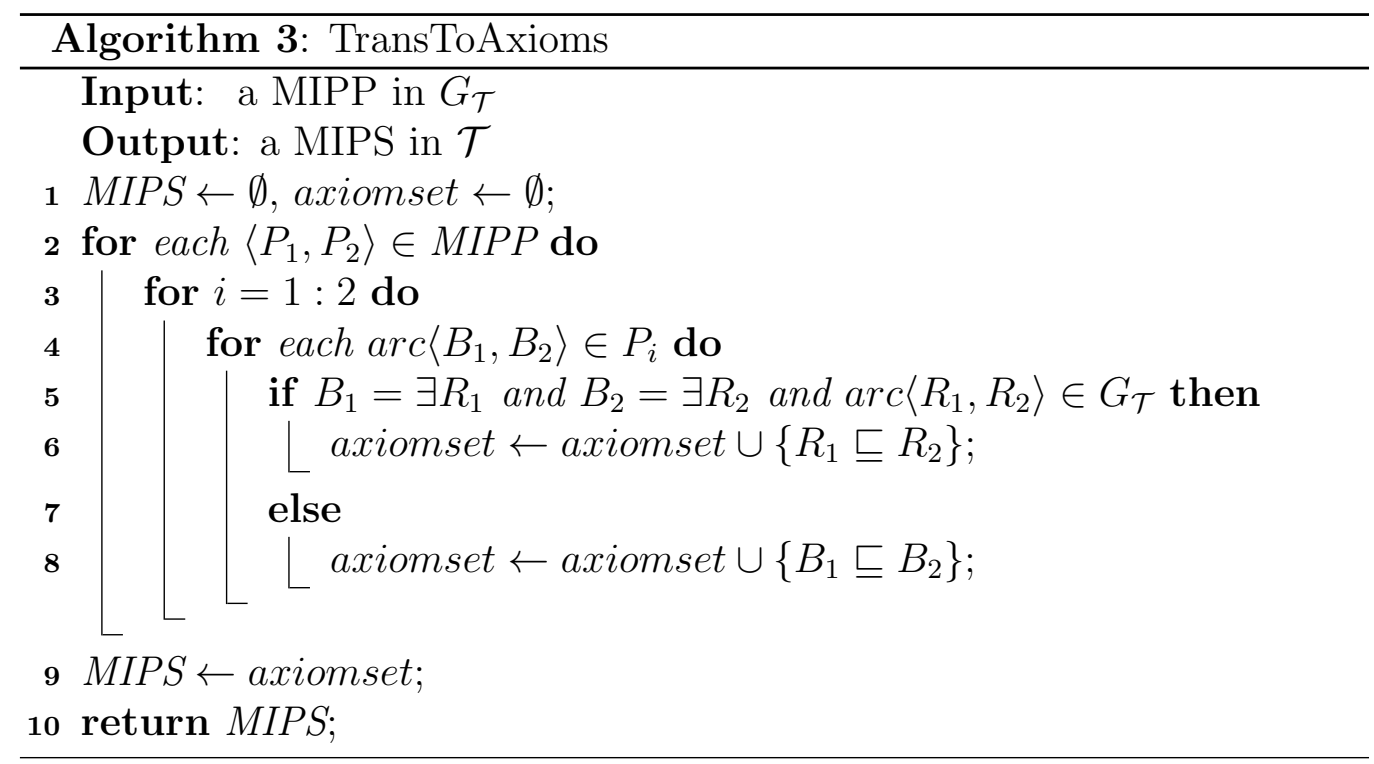

Algorithm 2, all of MIPP can be obtained in lines 7-8 which is nested in the third loop. From above analysis, Algorithm 2 is actually executed in a quadruple loop, but the complexity of function GetDisjointPathPairs which is invoked in line 6 of Algorithm 2 is in NP-hard. GetDisjointPathPairs aims to calculate all pairs of paths between the nodes $\neg A$ and $A$. Calculating a pair of paths is a traversal of the graph $G_{\mathcal{T}}$ to visit all nodes and edges in the graph, thus its complexity is $\mathrm{O}\left(|v|^{2}\right)$ in the worst case. Since there exists exponential number of pairs of paths in the worst case, there may be exponentially MIPP in the $G_{\mathcal{T}}$. It can be shown in the following example which is modified from Example 1 of [20].

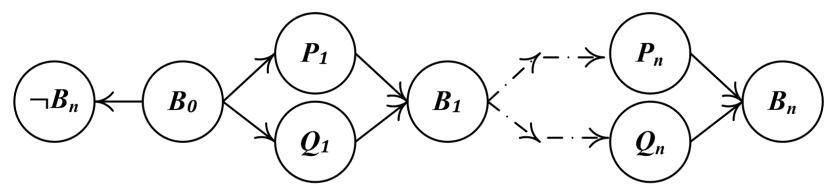

Figure 1: Directed graph in Example 4

Example 4. Given a TBox $\mathcal{T}$, where $\mathcal{T}=\left\{B_{0} \sqsubseteq \neg B_{n}, B_{i-1} \sqsubseteq P_{i}, B_{i-1} \sqsubseteq\right.$ $\left.Q_{i}, P_{i} \sqsubseteq B_{i}, Q_{i} \sqsubseteq B_{i} \mid 1 \leq i \leq n\right\}$. 
The TBox $\mathcal{T}$ can be transformed into a graph as shown in Figure 1. In Figure 1, we can obtain that the number of pairs of paths from $B_{0} \rightarrow \neg B_{n}$ and $B_{0} \rightarrow B_{n}$ is $2^{n}$. Therefore, the complexity of Algorithm 2 is in exponential time.

We continue to discuss Algorithm 1. Lines 3-4 of Algorithm 1 contain a single loop which can be executed in time $\mathrm{O}(n)$ ( $n$ is the size of mipps). From what we have discussed above, the complexity of Algorithm 1 is also in exponential time.

In Algorithm 3, a MIPP can be transformed into a MIPS. Lines 2-8 of Algorithm 3 contain a triplet loop which can be executed in cubic time. Therefore, the complexity of Algorithm 3 is $\mathrm{O}\left(n^{3}\right)$.

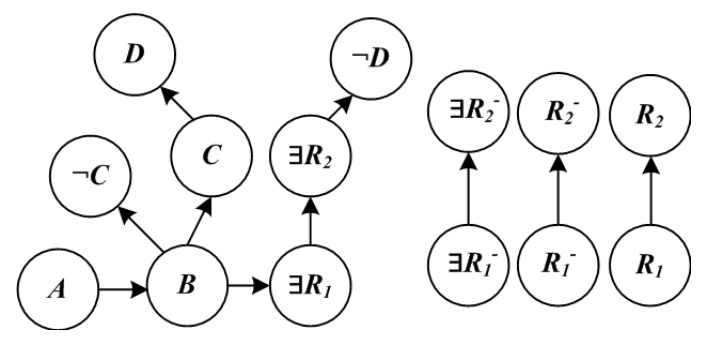

Figure 2: Directed graph in Example 5

Example 5. Given a TBox $\mathcal{T}$, where $\mathcal{T}=\{A \sqsubseteq B, B \sqsubseteq C, C \sqsubseteq D, B \sqsubseteq$ $\left.\neg C, R_{1} \sqsubseteq R_{2}, B \sqsubseteq \exists R_{1}, \exists R_{2} \sqsubseteq \neg D\right\}$.

According to the Construction Rules, we construct a directed graph as shown in Figure 2. In lines 2-6 of Algorithm 2, two MIPPs are identified in Fig.2, $\{B \rightarrow \neg C, B \rightarrow C\}$ and $\{B \rightarrow D, B \rightarrow \neg D\}$. In Algorithm 3, the path pair of MIPP will be transformed to axioms. For $\exists R_{1} \sqsubseteq \exists R_{2} \notin \mathcal{T}$ and $R_{1} \sqsubseteq R_{2} \models \exists R_{1} \sqsubseteq \exists R_{2}$, arc $\left(\exists R_{1}, \exists R_{2}\right)$ corresponds to $R_{1} \sqsubseteq R_{2}$ in $\mathcal{T}$. Finally, two MIPSs $\{B \sqsubseteq C, B \sqsubseteq \neg C\}$ and $\left\{B \sqsubseteq C, C \sqsubseteq D, R_{1} \sqsubseteq R_{2}, B \sqsubseteq\right.$ $\left.\exists R_{1}, \exists R_{2} \sqsubseteq \neg D\right\}$ are generated.

Theorem 9. Given an incoherent TBox $\mathcal{T}$, the output of Algorithm 1 is the set of all MIPSs of $\mathcal{T}$.

Proof. According to Theorem $5, n \in \operatorname{descendants}\left(S, G_{\mathcal{T}}\right)$ means $n \sqsubseteq S$. Thus, in lines 2-6 of Algorithm 2, $n_{1}=n_{2}$ means that there exists a path pair from the node $n_{1}$ (or $\left.n_{2}\right)$ to disjointness pair $(S, \neg S)$. By Definition 6 , these two paths are in a MIPP. Further, Algorithm 3 is applied to transform a MIPP to a MIPS. 
For any MIPS in $\mathcal{T}$, there exists a concept (or role) that is subsumed by two disjointness concepts (or roles). Thus, from Theorem 5, we can get two intersectional paths derived from two disjointness nodes in $G_{\mathcal{T}}$. Obviously, the intersectional point is the descendant of the two disjointness node. Namely, each MIPS can be calculated as shown in lines 1-4 of Algorithm 1. Therefore, the result of $\operatorname{Algorithm} 1$ is $\operatorname{mips}(\mathcal{T})$.

\subsection{Graph-based algorithms for TBox revision}

In this subsection, we give specific algorithms to instantiate the refinement revision operator defined in Section 3. Inspired by the works reported in [7], [8] and [21], we propose two algorithms, one is based on a scoring function, the other is based on a Hitting Set Tree (HS-Tree).

\subsubsection{Revision algorithm based on scoring function}

In [22], a scoring function is used to measure the inconsistency of an ontology. In [8], this scoring function is reformulated for ontology revision

in which axioms with the highest frequency of occurrence in $\operatorname{mips}_{\mathcal{T}_{2}}\left(\mathcal{T}_{1}\right)$ are picked. In the following, we introduce the definition of a scoring function.

Definition 15. Let $\mathcal{T}_{1}$ and $\mathcal{T}_{2}$ be two DL-Lite F $_{\mathcal{R}}$ TBoxes and $\mathcal{T}=\mathcal{T}_{1} \cup \mathcal{T}_{2}$. The scoring function for axiom $\alpha$ w.r.t $\mathcal{T}$, is a function $S_{\mathcal{T}}:$ mips $\mathcal{T}_{2}\left(\mathcal{T}_{1}\right) \mapsto N$ such that for all $\phi \in \bigcup_{\text {mips }}$

$$
S_{\mathcal{T}}(\phi)=\left|\mathcal{T}_{i} \in \operatorname{mips}_{\mathcal{T}_{2}}\left(\mathcal{T}_{1}\right): \phi \cap \mathcal{T}_{i} \neq \emptyset\right|
$$

Then, in order to define a specific refinement function, we modify the notion of axiom impact in [18], which evaluates the impact of an axiom when removing it from TBox.

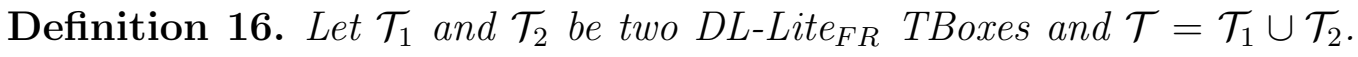
The impact of an axiom $\phi$, denoted by impact $(\phi)$, is defined as follows:

$\operatorname{impact}(\phi)=\left\{\begin{array}{l}0, \phi \notin \bigcup_{\text {mips }}, \\ \mid\left\{\phi^{\prime} \in \mathrm{cl}_{\mathcal{T}}(\phi) \mid \bigcup_{\text {mips }_{\phi}} \backslash\{\phi\} \cup\left\{\phi^{\prime}\right\} \text { is coherent. }\right\} \mid, \phi \in \bigcup_{\text {mips }} .\end{array}\right.$

In Definition 16, $\bigcup_{\text {mips }}=\bigcup_{\mathcal{T}_{i} \in \text { mips }_{\mathcal{T}_{2}}\left(\mathcal{T}_{1}\right)} \mathcal{T}_{i}$, mips $_{\phi}$ denotes the set of MIPS that contains the inclusion assertion $\phi$ and $\bigcup_{\text {mips }_{\phi}}=\bigcup_{\mathcal{T}_{i} \in \text { mips }_{\phi}} \mathcal{T}_{i}$. 


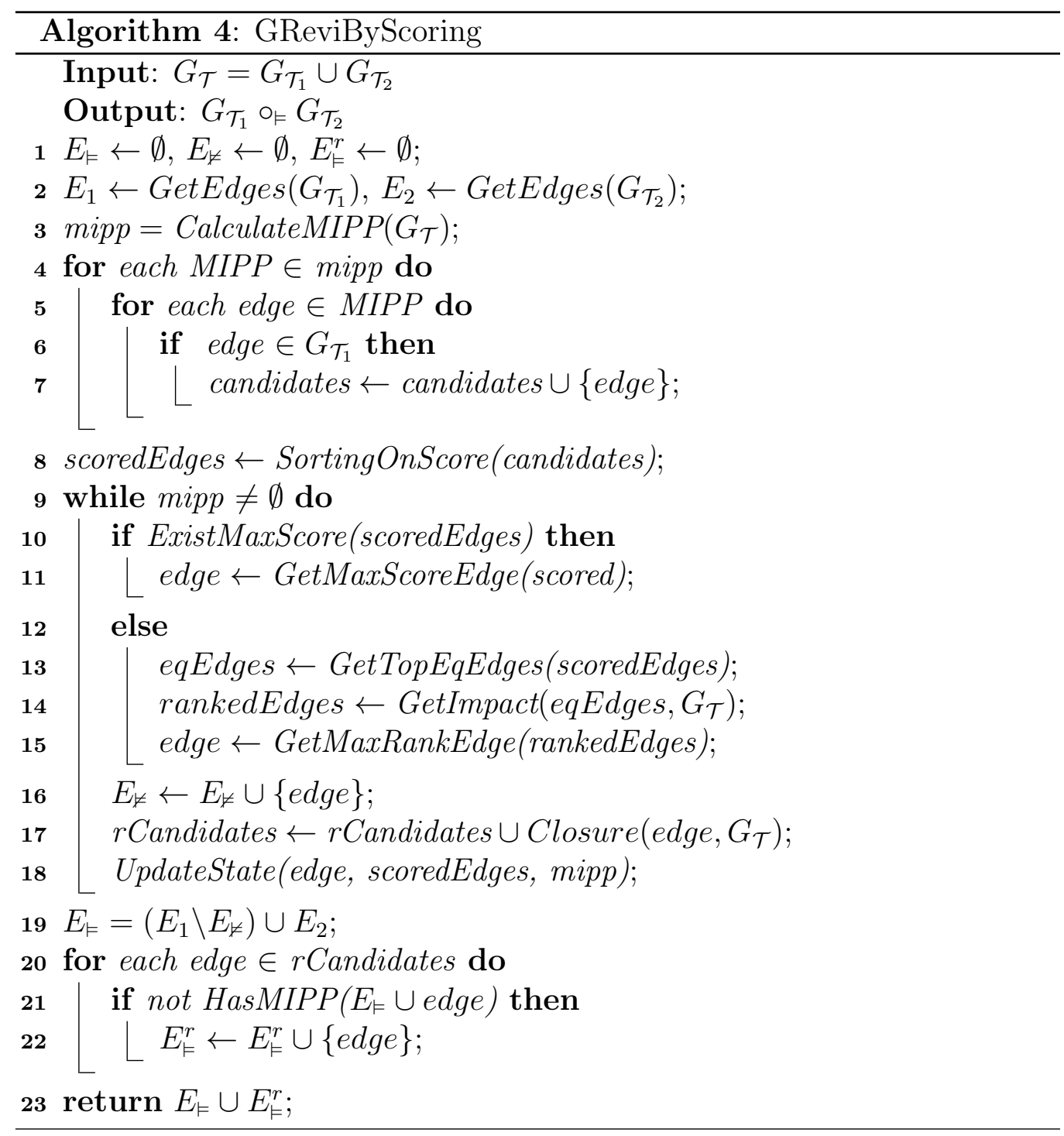


Based on the value of the scoring function, the incision function can select a candidate axiom $\phi$. In detail, an axiom will be selected if its score is maximum, otherwise, the impact value of these axioms will be calculated. Then, the axiom with minimum value of impact will be selected because the smaller the value of impact, the less number of axioms will be added.

By using the scoring function and the axiom impact, we present algorithm GReviByScoring (see Algorithm 4) for TBox revision, which consists of three steps.

Step 1 initialization. In lines $1-2$, the set $E_{1}$ (resp. $E_{2}$ ) is initially assigned by the set of edges of $G_{\mathcal{T}_{1}}$ (resp. $G_{\mathcal{T}_{2}}$ ). CalculateMIPP is invoked to obtain the set of MIPP and the returned result is assigned to mipp (line 3 ), and then the edges of mipp, which belong to $G_{\mathcal{T}_{1}}$, are selected to initialize the set candidates (lines 4-7). At the end of the step, the function SortingOnScore sorts candidates according to the frequency of the edges in mipp.

Step 2 calculating the unwanted set. In lines 9-15, the edge with maximum score is selected. The function GetTopEqEdges (line 13) groups the edges with the same maximum score in eqEdges. The function GetImpact is called to rank the impact values of the edges in eqEdge (line 14). The edge with minimum impact value is then selected and put in the unwanted set $\mathcal{T}_{\not \neq}$ (line 15). In line 17, the function Closure is called to compute the closure of $G_{\mathcal{T}}$, and the output is rCandidates. Finally, the function UpdateState removes the MIPPs that contain the selected edges.

Step 3 calculating the refinement set. The set $E_{\models}$ is first assigned with $\left(E_{1} \backslash E_{\nvdash}\right) \cup E_{2}$ ) (line 19). rCandidates is then scanned and checked to see whether a union of an edge in rCandidates with $E_{\not}$ leads to a new MIPP. All the edges that would not lead to new MIPPs are added in the refinement set $E_{\models}($ lines $20-22$ ).

In the following, we discuss the complexity of Algorithm 4. The exponential algorithm CalculateMIPP will be invoked in line 3 for calculating all the MIPP of $G_{\mathcal{T}}$. In lines 4-7, there exists a double loop. The times of the outer loop is the number of MIPPs and the times of the inner loop is the number of edges in corresponding MIPP. In line 8, a sorting algorithm will be invoked and its complexity is $\mathrm{O}\left(n^{2}\right)$ in the worst case. The function of line 9-18 is to calculate the unwanted set, which can be executed in polynomial time. Therefore, except for the calculation of MIPP, the complexity of Algorithm 4 is in PTIME. 
Example 6. Let $\mathcal{T}_{1}$ and $\mathcal{T}_{2}$ be two TBoxes. $\mathcal{T}_{1}=\{A \sqsubseteq B, B \sqsubseteq C, D \sqsubseteq$ $E, D \sqsubseteq F, F \sqsubseteq G\}, \mathcal{T}_{2}=\{A \sqsubseteq \neg C, A \sqsubseteq \neg B, C \sqsubseteq E, F \sqsubseteq \neg E\} . W e$ consider using algorithm GReviByScoring to revise $\mathcal{T}$.

We firstly encode $\mathcal{T}_{1}$ and $\mathcal{T}_{2}$ into the graph as shown in the left part of Figure 3 .
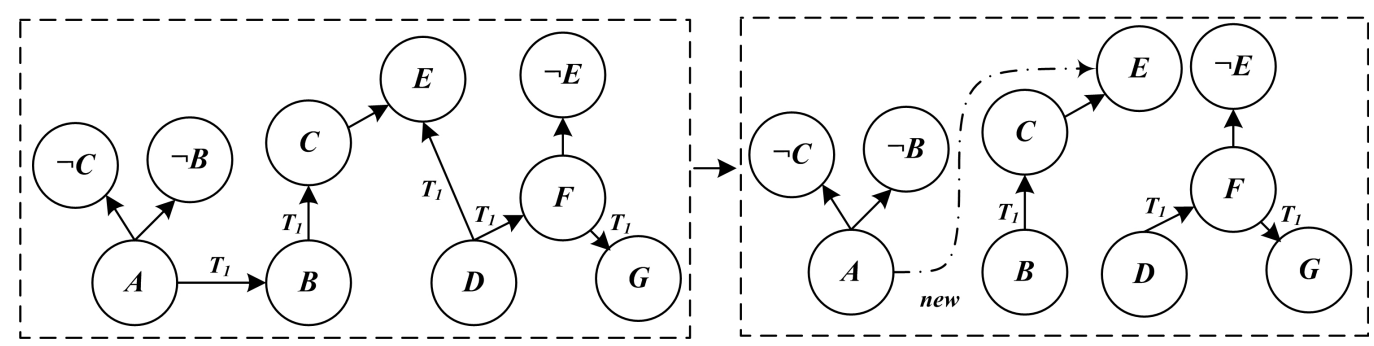

Figure 3: The process of revision in Example 6

According to Definition 6, we can obtain three MIPPs as follows: $\{A \rightarrow$ $\neg B, A \rightarrow B\},\{A \rightarrow B, B \rightarrow C, A \rightarrow \neg C\},\{D \rightarrow E, D \rightarrow F, F \rightarrow \neg E\}$. Based on lines 4-7, we obtain candidates $=\{A \rightarrow B, B \rightarrow C, D \rightarrow E, D \rightarrow$ $F\}$, whose edges are from $G_{\mathcal{T}_{1}}$.

In line 8 , the edges of candidates are sorted by the scoring function (see scoredEdges $=\{(A \rightarrow B, 2),(B \rightarrow C, 1),(D \rightarrow E, 1),(D \rightarrow F, 1)\})$.

At the first iteration of lines 9-15, the edge $(A \rightarrow B)$ is selected in lines 1011, which has the maximum value of scoring function. Then, edge $(A \rightarrow B)$ is appended in the set $E_{\not \neq}$ and the closure of $(A \rightarrow B)$, which is $\{A \rightarrow$ $C, A \rightarrow E\}$, is appended into the set $n$ Candidates. Then, the variables in the algorithm are updated. During the updating, the edge $(A \rightarrow B)$ is removed from candicates. The MIPPs including the edge $(A \rightarrow B)$ are removed from mipp and the scoredEdges is updated into $\{(D \rightarrow E, 1),(D \rightarrow F, 1)\}$. At the second iteration, there are two edges in candidates with the same value of scoring function. Therefore, the algorithm switches to "else case" , where the impact values are calculated for the edges, $(D \rightarrow E)$ and $(D \rightarrow F)$, based on lines 13-15. For the example, we have obtained that Closure $(D \rightarrow$ $\left.E, G_{\mathcal{T}}\right)=\emptyset$ and Closure $\left(D \rightarrow F, G_{\mathcal{T}}\right)=\{D \rightarrow G, D \rightarrow \neg E\}$. Further, the edge $(D \rightarrow F)$ is selected based on the function GetMaxRankEdge. Finally, when mipp turns into an empty set, the whole iteration finishes.

The above operation just gets unwanted set of edges from $G_{\mathcal{T}}$, there still has some available information in set $n$ Candidates. Thus, in lines 20-22, we 
retrieve the set $n$ Candidates and collect the edges in $n$ Candidates that will not lead to new MIPP. For the example, edge $(A \rightarrow C)$ is collected. The final result of the revision of example 6 is shown in the right part of Figure 3 .

\subsubsection{Revision Algorithm Based on Hitting Set Tree}

The incision function can be also implemented by a HS-Tree. HS-Tree is proposed in [23] where Reiter propose an approach that constructs a labeled tree based on a conflict set and reformulated in [7] for ontology debugging. During the process of ontology debugging, $\operatorname{mips}_{\mathcal{T}_{2}}\left(\mathcal{T}_{1}\right)$ can be regarded as a set of conflict sets. In a HS-Tree, nodes can be labeled with MIPS $\in \operatorname{mips}_{\mathcal{T}_{2}}\left(\mathcal{T}_{1}\right)$ and edges can be labeled with axioms $\alpha \in \bigcup_{\mathcal{T} \in \text { mips }_{\mathcal{T}_{2}}\left(\mathcal{T}_{1}\right)} \mathcal{T}$.

In the following, we propose the revision algorithm, GReviByHST (see Algorithm 5).

The algorithm also has three steps which are analogous to GReviByScoring. The main difference is in Step 2. In Step 2 of GReviByHST, the whole unwanted set $E_{\not}$ is obtained based on the method of hitting set tree. and the candicate set of unwant axioms are calculated once by invoking the function HS-Tree (line 8) and GetMinHS.

Next, we discuss the complexity of Algorithm 5. Algorithm 5 aims to perform the revision of ontologies by using a global optimal solution, which firstly calculates all the MIPP of $G_{\mathcal{T}}$ resembling to Algorithm 4. In line 3, the exponential algorithm CalculateMIPP will be invoked. Lines 4-7 contain a double loop which can be executed in square time. In line 8 , a function based on hitting set tree will be invoked. There exists exponential number of hitting sets [24] which can be executed in exponential time. There is no loop statement in lines $9-16$ which can be executed in polynomial time. Lines 17-19 contain a single loop which can be done in $\mathrm{O}(n)(n$ is the size of rCandidates). Therefore, except for the calculating of MIPP, the complexity of Algorithm 5 is also in exponential time.

Example 7. We consider using the TBoxes in Example 6 here. We perform the recision based on GRevisByHST.

GReviByHST firstly initializes the variables. According to lines 3-7, we get three $M I P S_{\mathcal{T}_{2}}\left(\mathcal{T}_{1}\right):\{A \rightarrow B\},\{A \rightarrow B, B \rightarrow C\},\{D \rightarrow E, D \rightarrow F\}$. According to the algorithm for calculating diagnoses using Hitting Set [7], we can build a hitting set tree, as shown in Figure 4. 


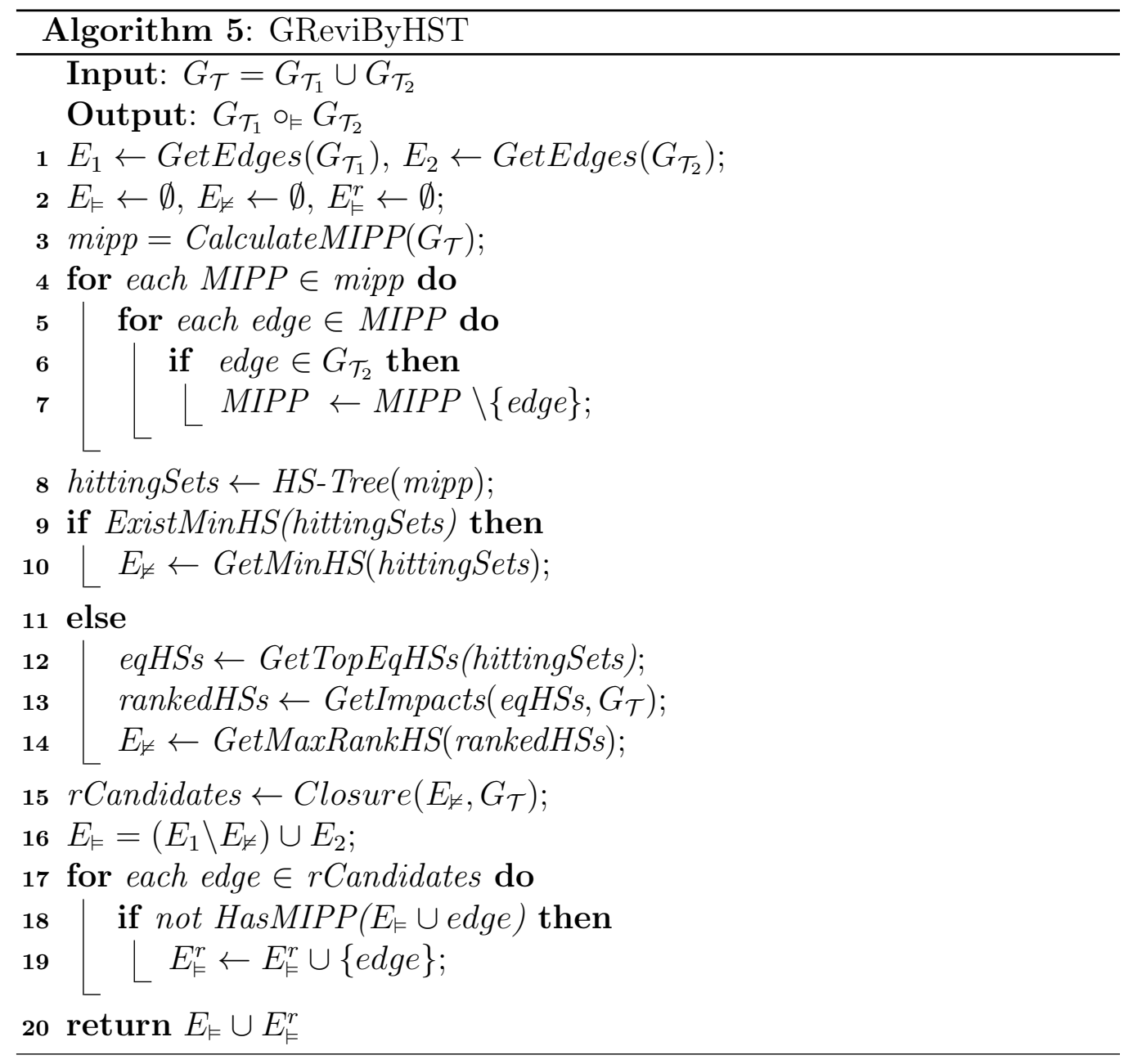


From Figure 4, we obtain two minimum hitting sets: $\{A \rightarrow B, D \rightarrow E\}$ and $\{A \rightarrow B, D \rightarrow F\}$. According to lines 9-14, function GetImpacts is called to calculate the impact value of these two sets. For $\{A \rightarrow B, D \rightarrow F\}$, the refinement set is $\{A \rightarrow C, A \rightarrow E, D \rightarrow \neg E\}$. For $\{A \rightarrow B, D \rightarrow E\}$, the refinement set is $\{A \rightarrow C, A \rightarrow E\}$. In order to guarantee minimal change, we choose the set $\{A \rightarrow B, D \rightarrow E\}$ as the unwanted set. Based on lines 17-19, the edges in rCandidates are added.

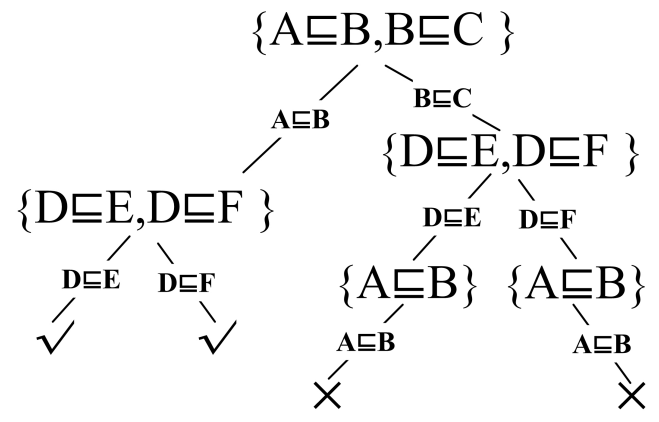

Figure 4: Hitting set tree of Example 7

\section{Implementation and evaluation}

We have implemented the debugging and revision algorithms presented in the previous section. The system is built on several open source software packages. We parse the structure of an ontology by OWLAPI ${ }^{1}$, which is a tool for managing OWL ontologies [25]. We transform an ontology into a graph and store it in the Neo4j graph database [26]. Neo4j ${ }^{2}$ is an open-source and high-performance graph database supported by Neo Technology[27]. The main ingredients of Neo4j are nodes and relationships. The nodes of Neo4j are used to record data in nodes which have user-defined properties and organized by relationships which also have user-defined properties. Furthermore, Neo4j provides a powerful declarative graph query language, cypher, which can be leveraged to querying and updating a graph database.

The ontologies used in our experiments have different sizes and structures. An ontology will be approximated [16], if it cannot be expressed in DL-Lite.

\footnotetext{
${ }^{1}$ http://owlapi.sourceforge.net

${ }^{2}$ http://www.neo4j.org/learn/cypher
} 
As we mentioned above, the incoherence appears in the merging process of two ontologies. To obtain the test ontologies, we partition an ontology into two different parts and modify them by inserting some "incoherentgenerating" axioms randomly, such as negative inclusions. For example, if $\mathcal{T}=\{A \sqsubseteq B, B \sqsubseteq C, B \sqsubseteq D\}$, it is obvious that $\mathcal{T}$ is coherent. To make it incoherent, we may insert $C \sqsubseteq \neg D$ into $\mathcal{T}$.

Table 1: Partitioned ontologies.

\begin{tabular}{|c|c|c|c|c|c|}
\hline \multicolumn{2}{|c}{ Ontology } & Axioms & NIs & Concepts & Roles \\
\hline \multirow{2}{*}{ AEO } & part 1 & 1043 & 1 & 225 & 7 \\
\cline { 2 - 6 } & part 2 & 1038 & 4 & 223 & 6 \\
\hline \multirow{2}{*}{ CL } & part 1 & 12373 & 19 & 2841 & 26 \\
\cline { 2 - 6 } & part 2 & 12365 & 20 & 2843 & 22 \\
\hline \multirow{2}{*}{ DOLCE } & part 1 & 294 & 5 & 34 & 66 \\
\cline { 2 - 6 } & part 2 & 335 & 13 & 33 & 70 \\
\hline \multirow{2}{*}{ Fly-Anatomy } & part 1 & 1726 & 34 & 285 & 41 \\
\cline { 2 - 6 } & part 2 & 1719 & 37 & 296 & 38 \\
\hline \multirow{2}{*}{ FMA } & part 1 & 16766 & 31 & 7091 & 27 \\
\cline { 2 - 6 } & part 2 & 16703 & 30 & 7093 & 31 \\
\cline { 2 - 6 } & part 1 & 274501 & 2 & 70339 & 15 \\
\hline \multirow{2}{*}{ GO } & part 1 & 272731 & 1 & 69968 & 14 \\
\cline { 2 - 6 } & part 2 & 51091 & 2 & 17302 & 1 \\
\hline \multirow{2}{*}{ Plant } & part 1 & 10984 & 24 & 1464 & 10 \\
\cline { 2 - 6 } & part 2 & 11029 & 2 & 1457 & 9 \\
\hline \multirow{2}{*}{ Terrorism } & part 1 & 1038 & 1 & 93 & 120 \\
\cline { 2 - 6 } & part 2 & 984 & 1 & 77 & 111 \\
\hline \multirow{2}{*}{ Transportation } & part 1 & 1415 & 158 & 387 & 79 \\
\cline { 2 - 6 } & part 2 & 1420 & 159 & 383 & 76 \\
\hline
\end{tabular}

In our work, we assume that the single ontology is consistent and the incoherence appears when ontologies are combined. In the preprocessing, we store the two ontologies into the Neo4j database, and use cypher query of neo4j to guarantee the precondition of our algorithms when adding a disjoint axiom to an ontology. The statistics of the used ontologies is given in Table 1.

All experiments are performed on a desktop computer with Intel Core i5$24003.1 \mathrm{GHz}$ CPU and 8GB of RAM, running Microsoft window 7 operating 
system, and Java 1.7 with $6 \mathrm{~GB}$ of heap space.

\subsection{Experiment of computing MIPSs based on graph}

In this paper, our approach of debugging aims to calculate MIPS. Many ontology reasoners can calculate MIPS. In this section, we performed comparative experiments with several popular reasoners, such as Pellet $^{3}$, Hermit $^{4}$, $\mathrm{FaCT}++^{5}, \mathrm{JFaCT}^{6}$.

We conducted the experiment from two aspects: one is to compare efficiency and the other is to compare performance on an ontology with different number of unsatisfiable concepts or roles.

Table 2: Conflict information in ontologies

\begin{tabular}{|c|c|c|c|c|c|c|c|c|c|c|}
\hline & AEO & CL & DOL. & Eco. & F.A. & FMA & GO & Pla. & Terr. & Tra. \\
\hline UC & 49 & 59 & 33 & 51 & 71 & 304 & 97 & 45 & 14 & 62 \\
\hline MIPS & 17 & 25 & 5 & 47 & 53 & 3 & 18 & 12 & 5 & 36 \\
\hline
\end{tabular}

Table 3: Time (in milliseconds) required to calculate all MIPS

\begin{tabular}{|c|c|c|c|c|c|}
\hline Ontology & Pellet & Hermit & FaCT++ & JFaCT & onGraph \\
\hline AEO & 1385 & 1986 & 814 & 1602 & 397 \\
\hline CL & 1536 & 2423 & 1187 & 2342 & 652 \\
\hline DOL. & 784 & 1121 & 515 & 867 & 362 \\
\hline Eco. & 1354 & 1429 & 677 & 1976 & 652 \\
\hline F.A. & 2129 & 3386 & 1926 & 4993 & 642 \\
\hline FMA & 47975 & 49867 & 45760 & 92017 & 3718 \\
\hline GO & 3805 & 5442 & 3893 & 9465 & 906 \\
\hline Pla. & 1654 & 2180 & 1102 & 1978 & 596 \\
\hline Terr. & 415 & 496 & 405 & 521 & 193 \\
\hline Tra. & 1892 & 2861 & 1457 & 4926 & 725 \\
\hline
\end{tabular}

The conflicting information is shown in Table 2 (we abbreviate the name of ontologies in table 1 and UC is the abbreviation of unsatisfiable concepts).

\footnotetext{
${ }^{3}$ https://github.com/complexible/pellet

${ }^{4}$ http://hermit-reasoner.com

${ }^{5}$ https://code.google.com/p/factplusplus

${ }^{6}$ http://jfact.sourceforge.net
} 
The experimental results are shown in Table 3. From Table 3(Graph-based Debugging approach is abbreviated as onGraph), our approach outperforms others. Especially, for some complex ontologies, such as FMA, our approach is significantly faster than others. Table 4 gives the result of scalability over the adapted GO ontology, with different number of unsatisfiable concepts or roles added. In the table, overflow means that the experimental program exhausts available memory.

We first identified all the disjoint concepts in GO ontology, and then increased the number of unsatisfiable concepts by adding common subsumed concept to the disjoint concepts randomly (we began with 100 unsatisfiable concepts for clearer comparison). The same treatment is also applied to roles. To have a better view, we transformed Table 4 into chart shown in Figure 5. From Figure 5, we can see that, with the growing number of unsatisfiable concepts and roles, the execution time of the state of the art approaches increases sharply. In comparison, the execution time of our approach grows moderately. The comparison result shows that our approach performs more steadily than other approaches in computing MIPS.

Table 4: Debugging time (in milliseconds) for incremental unsatisfiable concepts

\begin{tabular}{|c|c|c|c|c|c|}
\hline UC in GO & Pellet & Hermit & FaCT++ & JFact & onGraph \\
\hline 100 & 4381 & 6090 & 3991 & 8882 & 827 \\
\hline 110 & 4641 & 6280 & 4342 & 10864 & 912 \\
\hline 120 & 4982 & 7155 & 4948 & 12636 & 933 \\
\hline 130 & 6609 & 9771 & 6425 & 17174 & 1016 \\
\hline 140 & 8077 & 10132 & 7305 & 66663 & 1127 \\
\hline 150 & 9449 & 12082 & 8714 & overflow & 1209 \\
\hline 160 & 10210 & 13065 & 9630 & overflow & 1263 \\
\hline 200 & 12889 & 16573 & 12503 & overflow & 1769 \\
\hline
\end{tabular}

\subsection{Experiment of revising terminologies based on graph}

We conducted the experiment of revision to compare GReviByScoring and GReviByHST.

In this part, we set 30 minutes as the cut-off time. The experimental result of TBox revision is shown in Table 5. From Table 5, we can see that scoring-based algorithm outperformed HST-based algorithm. We can further see that, with the number of MIPP increasing, the execution time is also increasing and GReiviByHST runs slower than GReviByScoring. 


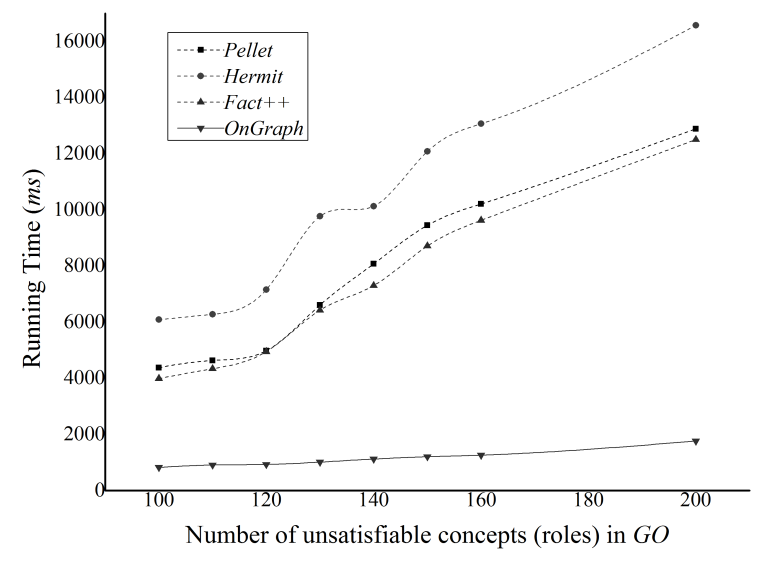

Figure 5: Time required to calculate MIPSs on GO

Table 5: Time (in seconds) required to ontology revision

\begin{tabular}{|c|c|c|c|c|c|}
\hline & AEO & CL & DOL. & Eco. & F.A. \\
\hline GReviByScoring & 14.076 & 16.649 & 2.358 & 20.198 & 20.452 \\
\hline GReviByHST & 21.972 & timeout & 4.776 & timeout & timeout \\
\hline & FMA & GO & Pla. & Terr. & Tra. \\
\hline GReviByScoring & 4.524 & 14.448 & 4.879 & 2.644 & 18.393 \\
\hline GReviByHST & 6.47 & 250.552 & 8.53 & 5.179 & timeout \\
\hline
\end{tabular}

The difference between the two algorithms is caused by the the specific incision function. GReviByHST is based on the method of hitting set tree to implement the incision function that can obtain the globally optimal solution. However, the complexity of finding minimal Hitting Sets is known to be NP-hard [24]. Thus, GReviByHST leads to a non-linear growth of running time. GReviByScoring employs a scoring function to define the incision function. Though it only achieves locally optimal solution, the complexity of calculating cardinality-minimal set is in PTime. Hence, the execution time of GReviByScoring is more than that of GReviByScoring.

The changes of the number of axioms in ontologies are collected in Table 6 and Table 7. As shown in Table 6 and Table 7, only on the ontology AEO, GReviByHST leads to fewer changes than that of GReviByScoring . 
Table 6: The change of ontologies in GReviByScoring

\begin{tabular}{|c|c|c|c|c|c|c|c|c|c|c|}
\hline & AEO & CL & DOL. & Eco. & F.A. & FMA & GO & Pla. & Terr. & Tra. \\
\hline Deleted & 6 & 12 & 3 & 27 & 3 & 1 & 7 & 3 & 5 & 14 \\
\hline Added & 6 & 22 & 6 & 144 & 35 & 1 & 13 & 3 & 5 & 66 \\
\hline
\end{tabular}

Table 7: The change of ontologies in GReviByHST

\begin{tabular}{|c|c|c|c|c|c|c|c|c|c|c|}
\hline & AEO & CL & DOL. & Eco. & F.A. & FMA & GO & Pla. & Terr. & Tra. \\
\hline Deleted & 5 & - & 3 & - & - & 1 & 7 & 3 & 5 & - \\
\hline Added & 5 & - & 6 & - & - & 1 & 13 & 3 & 5 & - \\
\hline
\end{tabular}

\section{Related work}

In the process of building and maintaining ontologies, new information may be inconsistent with ontologies when added it to the ontologies. Reasoning with inconsistent ontologies can obtain arbitrary conclusions. Therefore, inconsistency of ontology is an urgent problem that has to be resolved. This problem can be tackled by ontology debugging and revision which have been widely discussed in the literature.

First, we introduce the related work of ontology debugging. In general, the approaches for ontology debugging can be divided into two categories: glass-box approach and black-box approach. Glass-box approaches are based on reasoning algorithms and most of the glass-box approaches are obtained as extension of tableau-based algorithms for checking satisfiability of a DLbased ontology. The first tableau-based algorithm for ontology debugging is proposed in [14], but the algorithm is restricted to unfoldable $\mathcal{A L C}$ TBoxes. In [5], the tableau-based algorithm is extended to a more expressive DL, OWL DL . As pointed out in [21], one problem for the tableau-based algorithms is that some blocking techniques cannot be used. To avoid this problem, an automata-based approach is given in [28]. This approach has some nice theoretical advantage over tableau-based approaches. In order to compute MUPS, a debugging algorithm for DL-Lite ontologies is proposed based on the $\ln (\mathcal{T})$ which can be calculated off-line [29]. Our graph-based debugging algorithm belongs to the glass-box approach and specifically designed for DL-Lite ontologies. Furthermore, our algorithm can directly compute MIPS, whilst previous debugging algorithms are mostly applied to compute MUPS.

Different from the glass-box approaches, the black-box approaches do not need to modify the DL reasoner. However, in the worst case, the black-box 
approach may call the reasoner an exponential number of times. In [30], a general DL-based debugging approach is given. The approach is based on Reiter's algorithm and employs a simplified variant of QUICKXPLAIN[31] to generate the hitting set tree. According to [20], computing MUPS is a hard task for tractable DLs like $\mathcal{E} \mathcal{L}$. Therefore, it may be not possible to compute all MUPS for some large ontologies. In [32], the authors present an extensive evaluation of existing ontology debugging systems. However, they mainly evaluate systems for computing MUPS, but do not consider the computation of MIPS.

Next, we discuss the related work of ontology revision. Ontology revision deals with the incoherent problem when incorporating newly received information. In a nutshell, approaches to ontology revision can roughly be divided into two categories: syntax-based one and model-based one.

Most of practical revision operators are syntax dependant, i.e., if two logically dependant ontologies are revised by another ontology, the results of revision may not be logically equivalent. One type of syntax-based approaches is based on incision function. The incision function is used to select axioms, which are to be removed from the original ontology. The authors in [8] proposes a kernel revision operator and give two algorithms to define specific kernel revision operators. The authors in [9] develop a revision operator based on a trust-based incision function. The other type of formula-based approaches is to find maximally satisfiable terminologies. An algorithm is proposed in [33] to find maximally satisfiable terminologies for the description logic $\mathcal{A L C}$. A fine-grained approach is proposed in [34] in which notion of fine-grained repair is firstly introduced. In the approach, one axiom may be weakened to more axioms instead of being deleted.

Most of the works on model-based revision in DLs are devoted to proving the inexpressibility of model-based revision operators (see [35] and [36] for example). However, very few of them discuss the approximation of modelbased revision. One exception is the work in [37], where a revision operator is defined by a new semantics called features. However, feature-based revision also suffers from the inexpressibility problem and the algorithm is inefficient to deal with large ABoxes. Recently, there are some works on TBox revision based on a new semantics, called type semantics (see [38] and [39]). In [40], the authors propose an approach for approximating model-based revision operator by using a syntax-based revision operator. However, this work focuses on ABox revision instead of TBox revision. 


\section{Conclusion}

In this paper, we proposed graph-based approaches for debugging and revising incoherent DL-Lite ontologies.

In order to debug incoherent ontologies, we firstly encoded a DL-Lite ontology into a directed graph according to the given construction rules. Then, we gave a definition of MIPP on the graph and proposed the debugging approach based on MIPP. Finally, we conducted experiments over some adapted ontologies. Without the help of the DL reasoner, all the MIPS of an ontology can be calculated by backtracking some pairs of nodes in the graph. Our graph-based approach improves the ontology debugging both in efficiency and scalability.

In order to revise incoherent ontology, we propose the refinement revision operation based on a refinement revision space which can be obtained by a revision function. According to the principle of minimal change, the refinement revision operation can reserve more valid information. Finally, we gave two algorithms for ontology revision. one is based on incision function and the other is based on a HS-Tree. The experimental results shown that scoring-based algorithm leads to a better performance than HST- based algorithm, especially for the ontologies that contain numerous MIPSs.

In the paper, we focused on the family of DL-Lite. One of future works, we plan to extend our algorithms to more expressive description languages. As to DL-Lite, one of the remarkable characteristics is that it is suitable for query answering. The data complexity of answering unions of conjunctive queries in DL-Lite is polynomial in the size of the whole ontology and in LOGSPACE with respect to the size of ABox only [11]. As another of future work, we plan to study consistent query answering approaches based on graph.

\section{Acknowledgment}

We would like to thank the anonymous referees for their comments. Research presented in this paper was partially supported by National Science Foundation of China (No. 61272378), by the funds from JiangXi Educational Committee(No. GJJ12643) . 


\section{References}

[1] K. Amailef, J. Lu, Ontology-supported case-based reasoning approach for intelligent m-government emergency response services, Decision Support Systems 55 (1) (2013) 79-97.

[2] Y. Ma, B. Jin, Y. Feng, Dynamic evolutions based on ontologies, Knowl.Based Syst. 20 (1) (2007) 98-109.

[3] B. Parsia, E. Sirin, A. Kalyanpur, Debugging OWL ontologies, in: Proceedings of the 14th international conference on World Wide Web (WWW), 2005, pp. 633-640.

[4] G. Qi, F. Yang, A survey of revision approaches in description logics, in: Proceedings of 2nd International Conference on Web Reasoning and Rule Systems (RR), 2008, pp. 74-88.

[5] A. Kalyanpur, B. Parsia, E. Sirin, J. Hendler, Debugging unsatisfiable classes in OWL ontologies, J. Web Sem. 3 (4) (2005) 268-293.

[6] Q. Ji, Z. Gao, Z. Huang, M. Zhu, An efficient approach to debugging ontologies based on patterns, in: Proceedings of Joint International Semantic Technology Conference (JIST), 2011, pp. 425-433.

[7] S. Schlobach, Z. Huang, R. Cornet, F. Van Harmelen, Debugging incoherent terminologies, J. Autom. Reasoning. 39 (3) (2007) 317-349.

[8] G. Qi, P. Haase, Z. Huang, J. Z. Pan, A kernel revision operator for terminologies, in: Proceedings of the 21st International Workshop on Description Logics (DL), 2008, pp. 419-434.

[9] J. Golbeck, C. Halaschek-Wiener, Trust-based revision for expressive web syndication, J. Log. Comput. 19 (5) (2009) 771-790.

[10] C. E. Alchourrón, P. Gärdenfors, D. Makinson, On the logic of theory change: Partial meet contraction and revision functions, J. Symb. Log. 50 (2) (1985) 510-530.

[11] D. Calvanese, G. D. Giacomo, D. Lembo, M. Lenzerini, R. Rosati, Tractable reasoning and efficient query answering in description logics: The DL-Lite family, J. Autom. Reasoning. 39 (3) (2007) 385-429. 
[12] T. Venetis, G. Stoilos, G. B. Stamou, Query rewriting under query refinements, Knowl.-Based Syst. 56 (2014) 36-48.

[13] A. Artale, D. Calvanese, R. Kontchakov, M. Zakharyaschev, The DLLite family and relations, J. Artif. Intell. Res. 36 (1) (2009) 1-69.

[14] S. Schlobach, R. Cornet, Non-standard reasoning services for the debugging of description logic terminologies, in: Proceedings of the 13rd International Joint Conference on Artificial Intelligence (IJCAI), 2003, pp. 355-362.

[15] S. Gao, G. Qi, H. Wang, A new operator for ABox revision in DL-Lite, in: Proceedings of the 26th National Conference on Artificial Intelligence (AAAI), 2012, pp. 2423-2324.

[16] D. Lembo, V. Santarelli, D. F. Savo, Graph-based ontology classification in OWL 2 QL, in: Proceeding of 10th International Conference on The Semantic Web: Semantics and Big Data(ESWC), 2013, pp. 320-334.

[17] M. Akram, Bipolar fuzzy graphs with applications, Knowl.-Based Syst. 39 (2013) 1-8.

[18] N. Nikitina, S. Rudolph, B. Glimm, Interactive ontology revision, J. Web Sem. 12 (1) (2012) 118-130.

[19] M. Lenzerini, D. F. Savo, Updating inconsistent description logic knowledge bases, in: Proceeding of 20th European Conference on Artificial Intelligence (ECAI), 2012, pp. 516-521.

[20] F. Baader, R. Peñaloza, B. Suntisrivaraporn, Pinpointing in the description logic $\mathcal{E L}^{+}$, in: Proceeding of Advances in Artificial Intelligence (KI), 2007, pp. 52-67.

[21] A. Kalyanpur, B. Parsia, M. Horridge, E. Sirin, Finding all justifications of OWL DL entailments, in: Proceedings of 6th International Semantic Web Conference(ISWC) and 2nd Asian Semantic Web Conference (ASWC), 2007, pp. 267-280.

[22] G. Qi, A. Hunter, Measuring incoherence in description logic-based ontologies, in: Proceedings of 6th International Semantic Web Conference(ISWC) and 2nd Asian Semantic Web Conference (ASWC), 2007, pp. 381-394. 
[23] R. Reiter, A theory of diagnosis from first principles, Artif. Intell. 32 (1) (1987) 57-95.

[24] S. Schlobach, Diagnosing terminogies, in: Proceedings of the 20th National Conference on Artificial Intelligence (AAAI), 2005, pp. 670-675.

[25] M. A. Casteleiro, J. J. D. Diz, Clinical practice guidelines: A case study of combining OWL-S, OWL, and SWRL, Knowl.-Based Syst. 21 (3) (2008) 247-255.

[26] I. Robinson, J. Webber, E. Eifrem, Graph Databases, O'Reilly Media, Inc., 2013, pp. 25-63.

[27] J. Webber, A programmatic introduction to Neo4j, in: Proceedings of Conference on Systems, Programming, and Applications: Software for Humanity (SPLASH), 2012, pp. 217-218.

[28] F. Baader, R. Peñaloza, Automata-based axiom pinpointing, in: Proceeding of 4th International Joint Conference on Automated Reasoning (IJCAR), 2008, pp. 226-241.

[29] L. Zhou, H. Huang, G. Qi, Y. Qu, Q. ji, An algorithm for calculating minimal unsatisfiability-preserving subsets of ontology in DL-Lite, Journal of Computer Research and Development. 48 (3) (2011) 2334-2342.

[30] G. Friedrich, K. M. Shchekotykhin, A general diagnosis method for ontologies, in: Proceeding of 4th International Semantic Web Conference (ISWC), 2005, pp. 232-246.

[31] U. Junker, QuickXplain: preferred explanations and relaxations for overconstrained problems, in: Proceedings of the 18th National Conference on Artificial Intelligence (AAAI), 2004, pp. 167-172.

[32] Q. Ji, Z. Gao, Z. Huang, M. Zhu, Measuring effectiveness of ontology debugging systems, Knowl.-Based Syst. 71 (2014) 169-186.

[33] T. A. Meyer, K. Lee, R. Booth, J. Z. Pan, Finding maximally satisfiable terminologies for the description logic $\mathcal{A L C}$, in: Proceedings of the 21 th National Conference on Artificial Intelligence (AAAI), 2006, pp. 269274 . 
[34] J. Du, G. Qi, X. Fu, A practical fine-grained approach to resolving incoherent OWL 2 DL terminologies, in: Proceedings of the 23rd International Conference on Information and Knowledge Management (CIKM), 2014, pp. 919-928.

[35] G. Qi, J. Du, Model-based revision operators for terminologies in description logics., in: Proceedings of the 21st International Joint Conference on Artificial Intelligence (IJCAI), 2009, pp. 891-897.

[36] B. C. Grau, E. J. Ruiz, E. Kharlamov, D. Zhelenyakov, Ontology evolution under semantic constraints, in: Proceeding of 13th International Conference on Principles of Knowledge Representation and Reasoning (KR), 2012, pp. 137-147.

[37] Z. Wang, K. Wang, R. W. Topor, A new approach to knowledge base revision in DL-Lite, in: Proceeding of the 24th National Conference on Artificial Intelligence(AAAI), 2010, pp. 369-374.

[38] Z. Zhuang, Z. Wang, K. Wang, G. Qi, Contraction and revision over DL-Lite TBoxes, in: Proceedings of the 28th National Conference on Artificial Intelligence (AAAI), 2014, pp. 1149-1156.

[39] Z. Wang, K. Wang, Z. Zhuang, G. Qi, Instance-driven ontology evolution in DL-Lite, in: Proceedings of the 29th National Conference on Artificial Intelligence (AAAI), 2015, pp. 1656-1662.

[40] G. Qi, Z. Wang, K. Wang, X. Fu, Z. Zhuang, Approximating modelbased abox revision in DL-Lite: Theory and practice, in: Proceedings of the 29th National Conference on Artificial Intelligence (AAAI), 2015, pp. $254-260$. 